\title{
Ensemble Kalman Filter Data Assimilation for the Model for Prediction Across Scales (MPAS)
}

\author{
Soyoung Ha, Chris Snyder, William C. Skamarock, JefFrey Anderson, \\ AND NANCY COLLINS \\ National Center for Atmospheric Research, Boulder, Colorado
}

(Manuscript received 23 May 2017, in final form 5 September 2017)

\begin{abstract}
A global atmospheric analysis and forecast system is constructed based on the atmospheric component of the Model for Prediction Across Scales (MPAS-A) and the Data Assimilation Research Testbed (DART) ensemble Kalman filter. The system is constructed using the unstructured MPAS-A Voronoi (nominally hexagonal) mesh and thus facilitates multiscale analysis and forecasting without the need for developing new covariance models at different scales. Cycling experiments with the assimilation of real observations show that the global ensemble system is robust and reliable throughout a one-month period for both quasi-uniform and variable-resolution meshes. The variable-mesh assimilation system consistently provides higher-quality analyses than those from the coarse uniform mesh, in addition to the benefits of the higher-resolution forecasts, which leads to substantial improvements in 5-day forecasts. Using the fractions skill score, the spatial scale for skillful precipitation forecasts is evaluated over the high-resolution area of the variable-resolution mesh. Skill decreases more rapidly at smaller scales, but the variable mesh consistently outperforms the coarse uniform mesh in precipitation forecasts at all times and thresholds. Use of incremental analysis updates (IAU) greatly decreases high-frequency noise overall and improves the quality of EnKF analyses, particularly in the tropics. Important aspects of the system design related to the unstructured Voronoi mesh are also investigated, including algorithms for handling the C-grid staggered horizontal velocities.
\end{abstract}

\section{Introduction}

The development and application of variable-resolution global atmospheric models are areas of active research because these models achieve high resolution locally without the need for nested domains whose lateral boundary conditions are artificial and subject to unknown errors, and because the variable-resolution models are simpler compared to those that combine global and separate, regional models. The global nonhydrostatic Model for Prediction Across Scales (MPAS; Skamarock et al. 2012) provides horizontally variable-resolution using unstructured meshes, and also addresses the pole problems encountered in models using traditional latitude-longitude grids. We have developed a data assimilation system for MPAS that employs an ensemble Kalman filter (EnKF; Evensen 1994) as implemented in the Data Assimilation Research Testbed (DART; Anderson et al. 2009). This paper presents an overview of the assimilation system, with special attention to details of its implementation on

Corresponding author: Dr. Soyoung Ha, syha@ucar.edu the native MPAS unstructured mesh and a demonstration of its variable-resolution capabilities.

Data assimilation (DA) systems have been previously developed for a few other variable-resolution atmospheric models that employ stretching or cell division to locally enhance grid resolution (Laroche et al. 1999; Fox-Rabinovitz et al. 2002). While these systems utilize variable-resolution grids in their forecast model to calculate observation-forecast differences, the analysis increments are computed on separate uniform-resolution grids and then interpolated back to the native grids. The system presented here, in contrast, works entirely on the MPAS native mesh. The analysis thus retains the variable-resolution nature of the model forecasts.

A major challenge for data assimilation on variableresolution meshes is the need for estimating background error covariances on such meshes in a computationally efficient way. This difficulty is minimized in the EnKF because the required covariances are simply estimated from an ensemble of forecasts on the model's native mesh. Many assimilation schemes, however, employ correlation models whose computational 
implementation is tied in some fashion to logically rectangular or uniform-resolution grids. These issues will be discussed further in section 2.

The atmospheric component of MPAS (MPAS-A) uses spherical centroidal Voronoi meshes that enable global high-resolution simulations using either a quasiuniform- or a variable-resolution horizontal mesh configuration. The variable-resolution meshes allow for smooth transitions from coarse- to fine-resolution regions (Ringler et al. 2008; Ringler et al. 2010; Thuburn et al. 2009; Skamarock et al. 2012), unlike rectangular-grid regional models such as the Advanced Research Weather Research and Forecasting (WRF) Model (WRF-ARW; Skamarock et al. 2008) that use nesting with abrupt transitions in resolution and need artificial lateral boundaries. The MPAS mesh is also locally isotropic and conforming, in contrast to grids that are stretched or nested (Côté et al. 1993; Fox-Rabinovitz et al. 2002). For brevity, the quasiuniform and the variable-resolution meshes are simply described as the uniform and the variable meshes, respectively, throughout this paper.

MPAS uses a C-grid staggering of the prognostic variables in which the prognosed horizontal winds are the velocities normal to and located at the cell edges, and it is based on the work of Thuburn et al. (2009) and Ringler et al. (2010). In the assimilation, the horizontal winds are needed at arbitrary observation locations, and while approaches for handling the C-grid staggered horizontal velocities are clear for rectangular-grid models, there are certain subtleties that appear in the assimilation of the horizontal winds on unstructured $\mathrm{C}$-grid staggered meshes. We examine several options for the choice of wind variables within the analysis, for the reconstruction and interpolation methods needed for the wind observation operators, and for applying the analysis increments to update the winds in section 2.

In this paper we summarize the implementation and the performance of the system. We demonstrate that the MPAS-DART system is robust, reliable, and applicable to variable-resolution meshes as well as to quasiuniform meshes, by using retrospective studies that assimilate real observations for a one-month cycling period. We also examine the effect on forecast skill of using variable-resolution meshes in the cycling system, in addition to evaluating the effective scale at which forecasts become useful in the local refinement area of the variable-resolution mesh.

The quality of data assimilation in global atmospheric models is often degraded by spurious waves generated from dynamical imbalances arising from the analysis increments and subsequent adjustment. To reduce such spurious waves, we have implemented incremental analysis updates (IAU; Bloom et al. 1996) within MPAS.
Although IAU is not crucial to the performance of the system on variable-resolution meshes, it is helpful, especially in the tropics, and we briefly describe its implementation and impact.

The paper is organized as follows. Section 2 describes each component of the MPAS-DART system, discussing the observation operators for unstructured meshes in detail. The configurations of global ensemble cycling experiments for the retrospective case study are presented based on coarse uniform meshes in section 3 . Comparing to the performance over the coarse uniform mesh, the benefits of variable-resolution meshes are summarized during the cycles and extended forecasts in section 4, which also discusses its resolvable scales through the power spectra of ensemble analysis increments and precipitation forecasts. A summary and discussion is presented in section 5 .

\section{Analysis and forecast systems}

\section{a. The MPAS atmospheric solver}

The MPAS-A model solves the fully compressible nonhydrostatic equations using a horizontally unstructured spherical centroidal Voronoi mesh and it employs a C-grid staggering of the prognostic variables (Skamarock et al. 2012). The model uses a hybrid terrain-following height coordinate, described in Klemp (2011), which allows for the progressive smoothing of the coordinate surfaces with increasing height so as to remove smaller-scale terrain structure from the coordinate surfaces. The governing equations are cast in flux (or conservative) form, and the prognostic variables are dry-air density $\rho_{d}$, the horizontal momentum $\rho_{d} u$ and vertical momentum $\rho_{d} w$, the coupled potential temperature $\rho_{d} \theta$, and the coupled forms of moisture $\rho_{d} q_{v}$, cloud $\rho_{d} q_{c}$, and precipitation species.

On a horizontally unstructured C-grid centroidal Voronoi mesh, the horizontal momentum normal to the cell edge ( $u$ in Fig. 1a) is prognosed. Scalar variables such as potential temperature, dry-air density, and moisture are prognosed at the cell centers where they represent cell-averaged values in the finitevolume formulation. The prognosed vertical momentum is also C-grid staggered on the vertical cell faces located half a grid level above and below the cell centers. All other quantities are diagnosed from the prognostic variables (e.g., pressure). In particular, the zonal and meridional wind components at cell centers, which are needed for the physics parameterizations, are diagnosed from the normal velocities $u$ at all the edges of a given cell using a radial basis function (RBF) reconstruction (Bonaventura et al. 2011). 

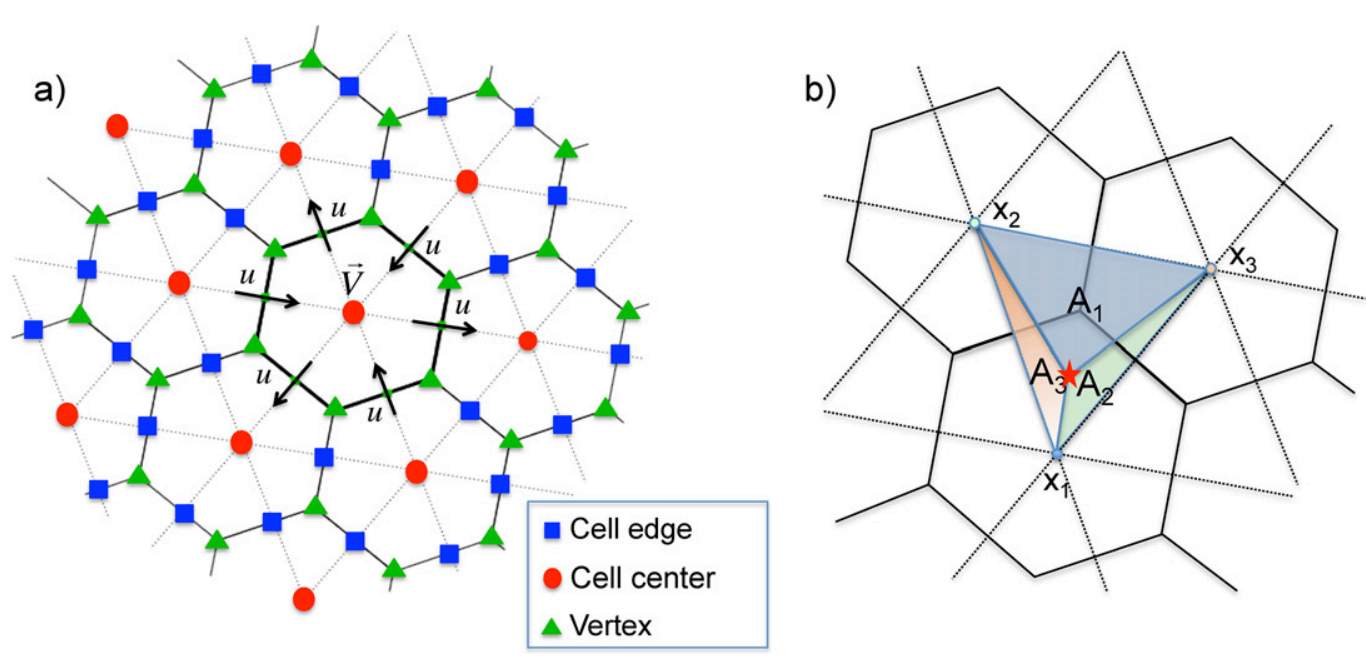

FIG. 1. Depiction of the horizontal MPAS grids that are constructed using an unstructured centroidal Voronoi tessellation. Primary cells are shown as hexagons in solid lines while the dual of the hexagonal mesh is marked as dotted triangles. (a) As illustrated, all scalar fields and reconstructed zonal and meridional winds are defined at the primary cell centers (red dots) and normal velocity $u$ is defined at cell edges (blue square). In MPAS-DART, vertices (green triangle) are used to search the triangle in the dual mesh that encloses an arbitrary observation point. (b) A barycentric interpolation to an observation point (red star) is depicted within the triangle.

The MPAS-A forecast model used in all experiments presented here is based on the release version 4 (http:// mpas-dev.github.io/), employing a physics suite adopted from the WRF Model. By default, it uses the "mesoscale_ reference" suite. This includes the Monin-Obukhov surface layer (Monin and Obukhov 1954), the Noah land surface model (Tewari et al. 2004), WRF singlemoment 6-class microphysics scheme (WSM6) (Hong and Lim 2006), Tiedtke cumulus (Tiedtke 1989), Yonsei University (YSU) planetary boundary layer (Hong et al. 2006), and the Rapid Radiative Transfer Model for GCM applications (RRTMG) longwave and shortwave radiation schemes (Iacono et al. 2008). For more details, see Skamarock et al. (2008). The model physics operate on cell-centered variables, and for the horizontal momentum they use the zonal and meridional wind components. Physics tendencies for the horizontal winds are produced at cell centers, and they are projected onto the edges to update the horizontal momentum. We also turn on the gravity wave drag over orography (GWDO) parameterization during the forecast, with the related static fields representing topography at corresponding grid resolutions. The static fields for the variable meshes are created based on Kim and Doyle (2005).

\section{b. The DART analysis system}

Data assimilation is performed by the EnKF as implemented in DART (http://www.image.ucar.edu/ DAReS/DART/). Speaking broadly, given observations and an ensemble of forecasts valid at the analysis time, the assimilation produces an ensemble of analyses whose mean and covariance approximate the Kalman filter update. The analysis ensemble then provides initial conditions for an ensemble of forecasts to the next analysis time. Readers are referred to Houtekamer and Zhang (2016) for a review of the ensemble Kalman filter for atmospheric data assimilation with an extensive list of references therein.

The specific variant of the EnKF that we employ is the ensemble adjustment Kalman filter (EAKF) of Anderson (2001); the relationship of the EAKF to other ensemble Kalman filter variants can be subtle, especially for nonlinear problems with finite precision arithmetic, and is discussed in Tippett et al. (2003). The EAKF employs serial processing of observations, that is, the ensemble analysis is performed for a single observation, then the resulting analysis ensemble is used as the background for the next observation, and so on until all observations valid at the analysis time are assimilated.

The application of EnKF to the MPAS variable mesh can be understood from the analysis equations. Because of the serial processing, it is sufficient to consider the analysis based on a single observation such as

$$
y=H(\mathbf{x})+\boldsymbol{\epsilon},
$$

where $\mathbf{x}$ is the state vector, $H(\cdot)$ is the (possibly nonlinear) observation operator relating $\mathbf{x}$ to the observed quantity, and $\boldsymbol{\epsilon}$ is a random observation error assumed to have zero mean and known variance $\sigma_{o}^{2}$. Letting superscripts $a$ and $b$ denote analysis and background 
quantities, respectively, and overbars signify an ensemble mean for an $N_{e}$-member ensemble, the EnKF update of the ensemble-mean background forecast $\overline{\mathbf{x}}^{b}$ given $y$ can be written as

$$
\overline{\mathbf{x}}^{a}=\overline{\mathbf{x}}^{b}+\mathbf{k}\left[y-\overline{H\left(\mathbf{x}^{b}\right)}\right],
$$

where $y^{b} \equiv H\left(\mathbf{x}^{b}\right)$ and the Kalman gain $\mathbf{k}$ is given by

$$
\mathbf{k}=\frac{\operatorname{cov}\left(\mathbf{x}^{b}, y^{b}\right)}{\sigma_{y}^{2}+\sigma_{o}^{2}}
$$

In addition, the update that produces each member's analysis $\mathbf{x}_{i}^{a}$, corresponding to the covariance update of the Kalman filter, is

$$
\mathbf{x}_{i}^{a}=\mathbf{x}_{i}^{b}+\beta \mathbf{k}\left[H\left(\mathbf{x}_{i}^{b}\right)-\overline{H\left(\mathbf{x}^{b}\right)}\right],
$$

where $\beta=\left[1+\sigma_{o}\left(\sigma_{y}^{2}+\sigma_{o}^{2}\right)^{-1 / 2}\right]^{-1}$ (Whitaker and Hamill 2002).

In the EnKF, the background error covariance between the state and observed variables, $\operatorname{cov}\left(\mathbf{x}^{b}, y^{b}\right)$, and the prior variance for the observed variable, $\sigma_{y}^{2}$, are sample estimates that are computed directly from the forecast ensemble:

$$
\begin{aligned}
\operatorname{cov}\left(\mathbf{x}^{b}, y^{b}\right) & =\frac{1}{N_{e}-1} \sum_{i=1}^{N_{e}}\left(\mathbf{x}_{i}^{b}-\overline{\mathbf{x}^{b}}\right)\left[H\left(\mathbf{x}_{i}^{b}\right)-\overline{H\left(\mathbf{x}^{b}\right)}\right], \\
\sigma_{y}^{2} & =\frac{1}{N_{e}-1} \sum_{i=1}^{N_{e}}\left[H\left(\mathbf{x}_{i}^{b}\right)-\overline{H\left(\mathbf{x}^{b}\right)}\right]\left[H\left(\mathbf{x}_{i}^{b}\right)-\overline{H\left(\mathbf{x}^{b}\right)}\right],
\end{aligned}
$$

where $\overline{\mathbf{x}^{b}}=1 / N_{e} \sum_{i=1}^{N_{e}} \mathbf{x}_{i}^{b}$ and $\overline{H\left(\mathbf{x}^{b}\right)}=1 / N_{e} \sum_{i=1}^{N_{e}} H\left(\mathbf{x}_{i}^{b}\right)$.

Therefore, applying Eqs. (1)-(5) on the native model mesh is straightforward regardless of the mesh's structure (i.e., each element of $\mathbf{x}^{b}$ resides on the native mesh). This enables EnKF to handle both the uniform- and variable-resolution MPAS meshes transparently once the observation operator $H$ is built on the native mesh. Nerger et al. (2007) and Du et al. (2016) also capitalize on the ease of implementation of the EnKF on variableresolution meshes, but in the case of finite-element ocean models.

Other covariance models, not based on sample estimates from a forecast ensemble, may be more difficult to apply to variable-resolution and unstructured meshes. For example, a simple way to build isotropic, homogeneous correlation models is to capitalize on the fact that they are diagonal in a spectral expansion (Laroche et al. 1999), but fast spectral transforms rely on regular grids. Other covariance models may be applicable on general meshes but more inconvenient to apply for variableresolution meshes (Fox-Rabinovitz et al. 2002). Finally, if the background correlation length scales are prescribed based on a fixed grid spacing, there is little to be gained from computing analysis increments directly on a variable-resolution mesh with locally higher resolution, as argued by Laroche et al. (1999). These factors, in our view, have led to a dearth of atmospheric data assimilation systems that utilize native variable-resolution meshes during the assimilation process.

The ensemble covariances used by the EnKF suffer from sampling error. To ameliorate its effects, we use localization applied to the observation-state covariance in Eq. (4), following Hamill et al. (2001) and Houtekamer and Mitchell (2001). Specifically, if $x_{i}$ and $k_{i}$ are the $i$ th elements of $\mathbf{x}$ and $\mathbf{k}$, respectively, covariance localization replaces $k_{i}$ by $\rho\left(d_{i}, L\right) k_{i}$, where $d_{i}$ is the distance between the locations of $x_{i}$ and the observation $y$, and $\rho\left(d_{i}, L\right)$ is the fifth-order polynomial, compactly supported correlation function of Gaspari and Cohn [1999, in their Eq. (4.10)] that is identically zero for $d_{i}$ greater than the localization radius $L$. We also employ inflation, in which the deviation of each member forecast from the ensemble mean is increased, before the assimilation step, by a spatially and temporally varying factor computed according to the scheme of Anderson (2009). Further specifics of the filter configuration will be discussed in section 3 a.

\section{c. An interface between MPAS and DART}

The MPAS-DART interface ${ }^{1}$ is composed of several routines to facilitate the communication between the analysis and the forecast system: 1) routines that read from and write to the MPAS restart files and translate to/from the analysis state vectors defined in DART; 2) observation (or forward) operators that interpolate the analysis state vectors to an arbitrary (e.g., observation) location of each observation type, including variable transformations when needed; and 3) a script that controls the integration of the MPAS model from one analysis time to the next. As described in section 2b, the entire analysis algorithm works directly with the unstructured mesh.

\section{1) ANALYSIS VARIABLES}

In MPAS-DART, the state variables updated by the EnKF algorithm are the uncoupled prognostic variables of the MPAS model such as potential temperature $\theta$, dry

\footnotetext{
${ }^{1}$ Here the term "interface" does not mean an online or direct coupling as used in other communities, but describes the framework that enables to exchange the information between the two components: the MPAS forecast model and the DART analysis system.
} 
density $\rho_{d}$, three-dimensional velocities, and all the hydrometeors $\left(q_{v}, q_{c}, q_{r}, q_{i}, q_{s}\right.$, and $\left.q_{g}\right)$. The analysis variables can be extended to include diagnostic variables, such as surface pressure. Observations of the diagnostic variables then affect the prognostic analysis variables through cross correlations, and the diagnostic fields are reset once the model is advanced from the analysis.

As the discrete state vector for the EnKF is represented directly on the native MPAS mesh, several approaches are considered in assimilating horizontal wind given that MPAS-A prognoses the horizontal momentum $u$ on and perpendicular to cell faces whereas the observed winds $\mathbf{V}$ are typically zonal and meridional wind components (see Fig. 1a). We will discuss the horizontal wind analysis approach in detail in the section $2 \mathrm{c}(3)$.

During the continuous analysis/forecast cycles, MPASDART is run in a restart mode carrying along all the physical tendencies and land surface states to make them consistent throughout the cycling period. Once the state variables are updated through the Kalman filter analysis, they are recoupled with the updated dry density $\rho_{d}$. The uncoupling of the MPAS prognostic variables for the analysis update, and the recoupling of the DART analysis variables as they are passed back to MPAS-A, take place in the interface routines. The coupling process is also described with an example in section $2 \mathrm{~d}$.

\section{2) OBSERVATION OPERATORS FOR SCALAR VARIABLES}

To compute prior observations (i.e., the prediction for observed variables based on the model forecasts), observation operators for all the analysis variables on the unstructured meshes are needed. Underlying all the observation operators is an interpolation from the unstructured centroidal Voronoi mesh to an observation location. To accomplish this interpolation in the horizontal, we employ a barycentric (e.g., area weighted) interpolation that uses the Delauney triangular mesh, which is the dual of the Voronoi mesh, as depicted in Fig. 1b. Note that the cell centers in the Voronoi mesh are located at the vertices of the triangular mesh. To interpolate to an observation point, the dual-mesh triangle containing the observation point is first identified by finding the vertex of the Voronoi mesh closest to that point. The interpolated value $\hat{x}$ is then given as

$$
\hat{x}=\frac{\sum_{i=1}^{3} A_{i} x_{i}}{\sum_{i=1}^{3} A_{i}},
$$

where $A_{i}$ is the area of each triangle $i$, and $x_{i}$ are the scalar values at the Voronoi cell centers (triangle vertices) as shown in Fig. 1b. In this algorithm, all the grid points are treated in a three-dimensional Cartesian coordinate with the origin at the center of the earth, thus avoiding singularity issues at the poles. We also note that the interpolation is continuous across the triangle faces. Any vertical interpolation takes place before horizontal interpolation [i.e., values at the triangle vertices are interpolated to constant-height surfaces before the barycentric interpolation in Eq. (6) is applied]. When the observed variable is different from the analysis variable (e.g., temperature observations for the update of potential temperature in the analysis), the spatial interpolation is first applied to the input variables (such as potential temperature, dry density, and water vapor mixing ratio) individually, then the observed variable is computed from those fields interpolated at the observed location. For surface observations, the height difference between the observation site and the model terrain height is checked, and if the discrepancy is larger than a predefined threshold (which is $100 \mathrm{~m}$ in the current study), the observation is rejected.

\section{3) OBSERVATION OPERATORS FOR HORIZONTAL WIND}

In the horizontal C-grid discretization used in MPAS, normal velocities $u$ are prognosed at cell edges. As the horizontal winds are typically observed as zonal and meridional winds, we need a variable transformation as well as a spatial transformation in the observation operator $H$ to compute the prior observations for horizontal wind. We have examined several options that differ in their choice of the wind variable for the analysis state vector, the horizontal interpolation method, and how the analysis increments are applied to update the wind analysis field for the model integration. We discuss two main options here. The first approach is to use the reconstructed zonal and meridional winds at cell centers along with barycentric interpolation in the observation operator, followed by projecting the analysis increments in the horizontal winds onto the normal velocities at cell edges. This approach is analogous to that used by MPAS-A model physics for the horizontal winds. Alternatively, we can directly use the MPAS prognostic normal velocities in the observation operator by reconstructing zonal and meridional wind components at the observation locations using the RBF. In this case, the MPAS prognostic normal velocities are used as analysis variables and the wind field is updated directly through the analysis (as opposed to the incremental update in the first approach). For reconstructing the velocity at an observation point, while the barycentric interpolation in the first approach is always applied using the values from three Voronoi cell centers (that are located at the 
vertices of the dual-mesh triangle), the RBF approach can make use of as many edges as users choose. Our test of the RBF approach in DART indicate that using too few points in the RBFs can result in distinct discontinuities between the nearby prior observations (the interpolation is not continuous), and using a large stencil with too many points may lead to smoothing the values excessively and is computationally expensive. A moredetailed discussion of velocity reconstruction on the Voronoi mesh is given by Peixoto and Barros (2014). We compare the two approaches in the cycling context in section 3 .

\section{d. Incremental analysis updates (IAU)}

In the EnKF analysis, covariance localization and inflation are typically used to ameliorate the effect of spurious correlations due to the limited ensemble size, but they also produce dynamical imbalances and inconsistencies between the state variables in the background ensemble covariance (Mitchell et al. 2002; Houtekamer and Mitchell 2005; Kepert 2009; Greybush et al. 2011). When the forecast model is initialized from the analysis, it can experience spurious oscillations driven by imbalances introduced in the analysis step. Within regional models, spurious waves (noise) generated by imbalanced initial conditions are absorbed by the sponge layer at the lateral boundaries as the waves propagate out of the domain. But the spurious waves in global models can contaminate solutions for much longer periods until they are damped out by model filters. To deal with such noise and render the analysis more dynamically consistent, the IAU is widely employed as a means of distributing analysis increments over a certain time window (Bloom et al. 1996; Zhu et al. 2003; Polavarapu et al. 2004, and many more).

We implemented IAU within MPAS as follows. As pointed out in section $2 \mathrm{c}$, the continuous prognostic equations in MPAS are cast in flux form while the EnKF analysis vector consists of uncoupled variables including dry density $\rho_{d}$. Therefore, when analysis increments for uncoupled variables are applied to the model, they need to be first "recoupled" with the updated dry density. Let us consider potential temperature $\theta$, for example. After the analysis is complete at time $t$, the analysis increment $\left(\delta \theta=\theta^{a}-\theta^{b}\right)$ is multiplied by the updated dry density $\rho_{d}$ to give an increment $\left[\delta\left(\rho_{d} \theta\right)=\theta \delta \rho_{d}+\rho_{d} \delta \theta\right]$ for the corresponding coupled variables. Assuming a constant forcing over the time window $(\Delta \tau)$ centered on the analysis time over which IAU affects the model integration, the analysis increments for the coupled variables $\delta\left(\rho_{d} \theta\right)$ are then divided by the number of time steps within the window $(n=\Delta \tau / \Delta t)$ and incorporated into the tendency every time step when MPAS is integrated from $t-\Delta \tau / 2$. In this study, $\Delta \tau=6 \mathrm{~h}$, followed by a free forecast for $3 \mathrm{~h}$ to the next analysis time.

Recent studies have introduced four-dimensional IAU to consider the propagation of the analysis increments with more sophisticated time filtering within the assimilation window (Lorenc et al. 2015; Buehner et al. 2015; Lei and Whitaker 2016). We implemented the IAU to make further extension to include the timevarying increments straightforward, but for simplicity we only consider the IAU with the constant forcing here.

\section{Cycling experiments}

As the MPAS-DART system is built on the native coordinate of the MPAS model, it is immediately applicable for variable-resolution MPAS meshes and various grid resolutions. To demonstrate that the MPAS-DART system runs reliably and performs well on both uniform and variable meshes, global ensemble cycling experiments are conducted on two different meshes. In this section, we describe the details of the experiment setup and present the results over the coarse uniform mesh on which the configurations for MPAS-DART are determined.

\section{a. Experiment design}

In the retrospective case study presented here, the global analysis/forecast ensemble systems are configured on 120-km uniform (with total of 40962 cells) and $120-30-\mathrm{km}$ variable meshes (with total of 133890 cells), respectively. Figure 2 depicts the variable mesh with the high-resolution region (i.e., the $30-\mathrm{km}$ grid spacing region) covering the contiguous United States (CONUS). A transition zone with the width of roughly $3000 \mathrm{~km}$ separates the high-resolution region from the lowresolution, $120-\mathrm{km}$ mesh for the rest of the globe. In each experiment, both analysis and forecast ensemble systems are configured on the same MPAS mesh and use 56 vertical levels up to $30 \mathrm{~km}(\sim 10 \mathrm{hPa})$.

In the cycling experiments, a 96-member ensemble is used for a 1-month period from 0000 UTC 25 May 2012 cycling every $6 \mathrm{~h}$. To construct the initial ensemble for global cycling experiments, independent random draws from a Gaussian distribution with tiny standard deviation $^{2}$ are added to a first guess [which is the NCEP Final Operational Global Analysis (FNL) data in this study] valid at 15 May 2012, from which the model is freely run for 10 days. The 10-day ensemble forecasts are then recentered on the FNL analysis at the initial cycle (e.g., 0000 UTC 25 May 2012). This initial ensemble is

\footnotetext{
${ }^{2}$ The amplitude of random noise is 0.0001 multiplied by each state vector.
} 

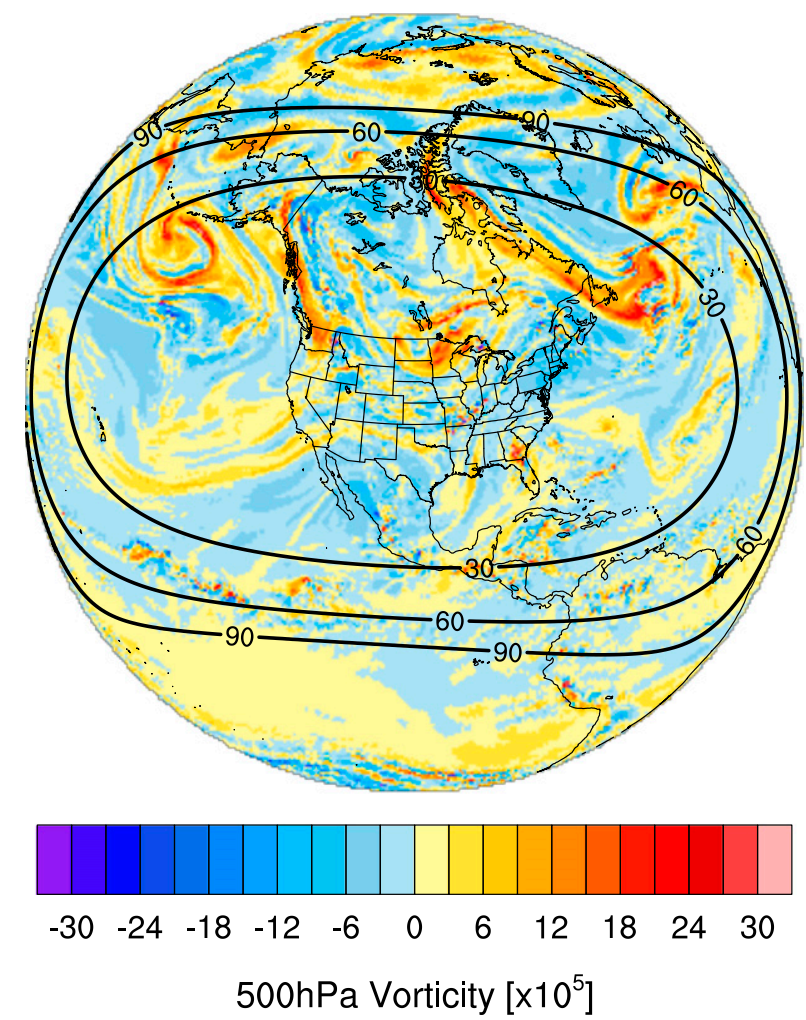

FIG. 2. Grid resolutions in $120-30-\mathrm{km}$ variable mesh (named " $\mathrm{x} 4$ "), contouring every $30 \mathrm{~km}$ in solid lines, superimposed over relative vorticity at $500 \mathrm{hPa}$ (colored) at 36 -h forecast valid at 1200 UTC 29 May 2012.

first generated on the $120-\mathrm{km}$ uniform mesh, then horizontally regridded to the $120-30-\mathrm{km}$ variable mesh using the barycentric interpolation. As such, both meshes can be cycled from the same initial ensemble forecasts. Throughout the paper, the experiments on the $120-\mathrm{km}$ coarse uniform and the $120-30-\mathrm{km}$ variable meshes are also called " $\mathrm{x} 1$ " and " $\mathrm{x} 4$ " respectively.

\section{b. Model and filter configurations}

The model configuration is the same between the two different mesh experiments, except for the RungeKutta time step $\Delta t$, which is based on the finest grid spacing $\Delta x$ in each grid mesh. In this study, the $120-\mathrm{km}$ uniform and the $120-30-\mathrm{km}$ variable meshes use the integration time step $\Delta t$ as 600 and $150 \mathrm{~s}$, respectively.

The analysis variables used in these experiments are potential temperature, dry density, horizontal wind, vertical velocity, all the hydrometeors, surface pressure, precipitable water, $10-\mathrm{m} u$ - and $v$-wind components, 2-m temperature, potential temperature, and water vapor mixing ratio.

In the EnKF analysis, the impact of each observation is localized by a function of the distance between the observation and state variables, according to the compactly supported correlation function of Gaspari and Cohn (1999). The radius of the ellipsoidal covariance localization function is set to $1200 \mathrm{~km}$ in the horizontal direction and $6 \mathrm{~km}$ in height in all the experiments. Different localization radii were tested in various vertical coordinates (such as pressure or scale height) in the cycling context, but overall the results were not significantly distinguishable, especially in terms of 5-day forecasts from the EnKF mean analyses. In our global cycling experiments, the localization generally affected the stability of the ensemble system more than the analysis quality per se. It was also found that a proper localization radius might be different in different regions (e.g., Northern vs Southern Hemisphere or troposphere vs stratosphere) due to the inhomogeneous global observing network and differences in the relevant dynamics. Therefore, we do not optimize individual experiments but demonstrate that the same filter design can be applied to different grid configurations in this study.

To better deal with the sampling error in the 96-member ensemble, the prior covariance is inflated before observation operators are applied every cycle, and the coefficient is spatially and temporally adaptive to heterogeneous observations and the systematic error in prior covariance estimates (Anderson 2007, 2009, 2012). In this study, initial mean and standard deviation of the inflation factor is 1.0 and 0.6 , respectively, and the inflation mean value is damped by $10 \%$ before the next assimilation cycle.

\section{c. Observations}

In the cycling experiments, the same real observations are assimilated in both meshes for the 1-month period. We assimilate all the observations available in the operational data stream for the NCEP Global Data Assimilation System (GDAS) except for satellite radiances. Included are radiosonde soundings (raob), Aircraft Communication, Addressing, and Reporting System (ACARS), marine (MARINE), meteorological terminal aviation routine weather report (METAR) and other land surface observations (SFC), satellite winds (SATWND), GPS radio occultation (GPSRO) refractivity data, and GPS precipitable water (GPSPW). A sample observing network valid at 1200 UTC 1 June 2012 is plotted in Fig. 3. All the observations available within a window of $\pm 1.5 \mathrm{~h}$ around each analysis time are assimilated.

The assimilation system utilizes observations directly from the NCEP GDAS files. As part of observation preprocessing, we implemented a data compression capability for ACARS and satellite winds (by averaging such dense observations within each grid cell to produce 


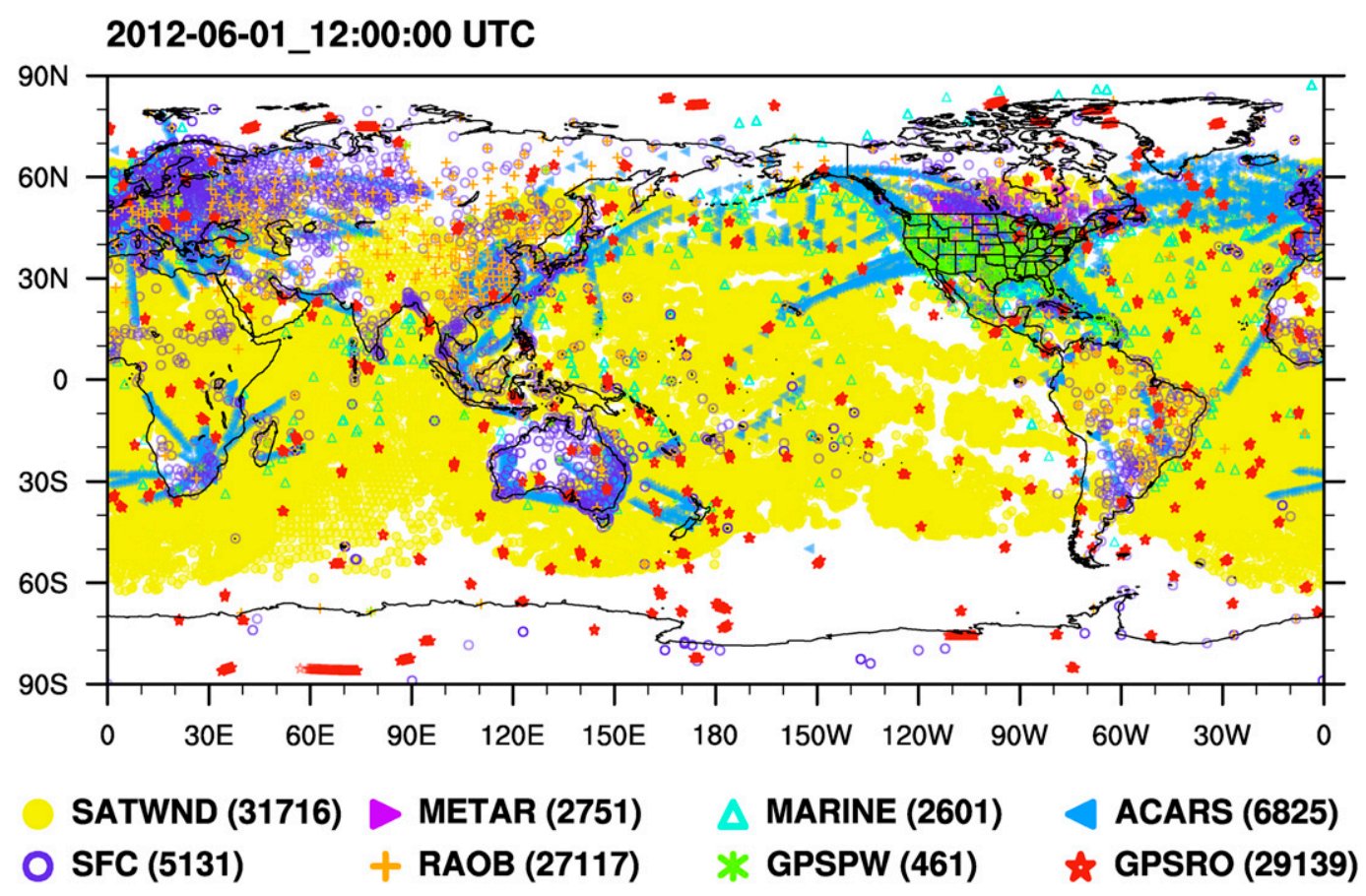

FIG. 3. Sample observing network used in cycling experiments, valid at 1200 UTC 1 Jun 2012. A total number of observations is shown in the parentheses of each observation type at the bottom.

superobs) in MPAS-DART, but the impact of superobing those data was mostly insignificant in our test experiments. Meanwhile, the GPSRO data provide excessively high vertical resolutions for our 56-level MPAS model, especially in the upper-level atmosphere, so we retain GPSRO data only every $200 \mathrm{~m}$ in the vertical.

Observation errors are assumed independent between different observation types and between different observations of a given type. Observation error standard deviations are specified as in the NCEP GDAS, except for the relative humidity observations for which the standard deviation is reduced from $20 \%$ to $10 \%$.

From the DART point of view, one of the biggest changes in treatment of observations is to assimilate the refractivity data from all the available GPS satellites using the same observation error variance as in the NCEP Gridpoint Statistical Interpolation analysis system (GSI) (Cucurull 2010). The original (or old) observation errors were defined based on Kuo et al. (2004). Figure 4a illustrates the old and new refractivity observation errors in extratropics and tropics, respectively, and Figs. $4 \mathrm{~b}-\mathrm{d}$ show the resulting bias error of 6 -h forecast with respect to the refractivity data when the GPSRO data are assimilated with those errors during the cycles. Here, tropics range from $20^{\circ} \mathrm{S}$ to $20^{\circ} \mathrm{N}$ including the gridresolution transition zone. In the new observation error specification, the absolute error is reduced up to a factor of 10 (e.g., from 0.3 to 0.03 at $17 \mathrm{~km}$ over the tropics), leading to a significant bias reduction throughout the entire atmosphere in all different regions. With the GPSRO observation error adjustment in the analysis, the forecasts are consistently improved in all other variables both in rms and bias error. The largest positive impact is observed in temperature in the upper atmosphere over the tropics (not shown). Because of the quality of GPSRO retrievals, we discard all the refractivity data below $3 \mathrm{~km}$ in the experiments.

\section{d. Wind data assimilation}

We compare two different wind data assimilation methods introduced in section $2 \mathrm{c}(3)$ in the ensemble cycling context. Here the first method uses the reconstructed winds at cell centers in the observation operator and is called "cell_wind," and the second approach uses the normal velocity at edges, and is named "edge_wind." For the comparison between the two methods, we use normal velocity from edges comprising the same three cells so that the wind correlations in the background error covariances are computed from the same set of edges in both cases. Except for the way horizontal wind observations are assimilated and updated, all other configurations for the analysis and the forecast are identical. 

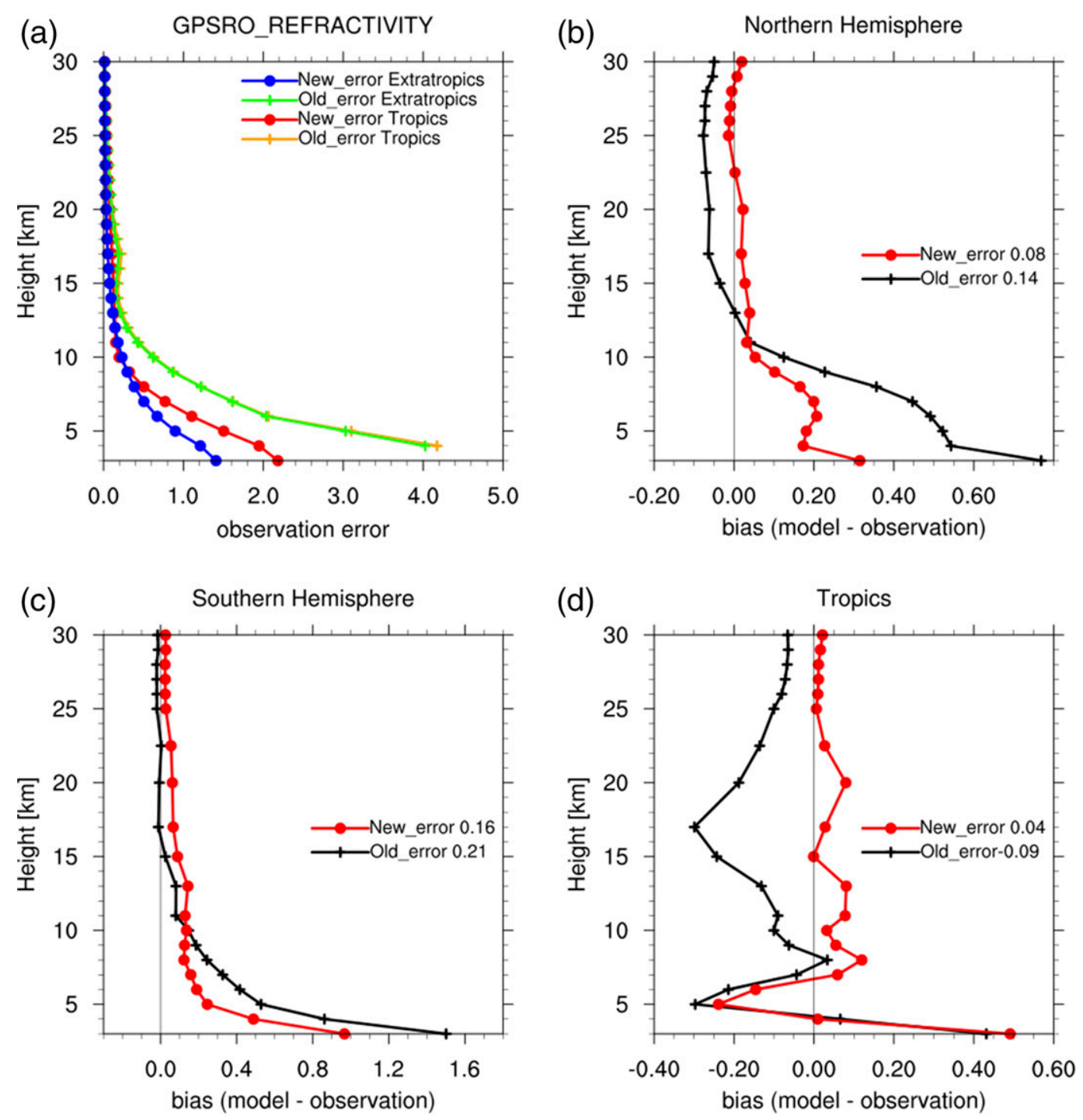

FIG. 4. (a) Vertical profile of two different refractivity observation errors assigned to GPSRO observations during the assimilation over tropics and extratropics, respectively, and domain- and cycle-averaged bias error of 6-h forecast (prior) with respect to GPS refractivity observations that are assimilated using those different observation error variances over the (b) Northern Hemisphere, (c) Southern Hemisphere, and (d) tropics. Forecast bias errors averaged over all levels are written next to the corresponding experiment names in (b)-(d).

Figure 5 depicts the root-mean-square (rms) fit of 6-h ensemble-mean forecasts to zonal-wind sounding observations. As shown in the time series, the 6-h forecasts from the ensemble mean analyses using the reconstructed cell-center winds as analysis variables consistently outperform the ones using the normal velocities on edges. That is consistent throughout the entire atmosphere, as averaged over the entire globe in the vertical profile. Verification for the meridional wind component produces a similar result while the forecast fits to radiosonde temperature show little difference between the two experiments (not shown). Based on this test, we employ reconstructed winds at cell centers as analysis variables for winds in all the following experiments presented in this paper.

\section{e. The effect of IAU}

In the global analysis, it is critical to effectively suppress high-frequency oscillations for the numerical stability and the quality of subsequent forecasts. To examine the level of such noise, a time series of surface pressure tendencies $\left(\mathrm{Pa} \mathrm{s}^{-1}\right)$ as an area-weighted, globalaveraged absolute value is shown for MPAS forecasts from different initial conditions over the 120-km uniform mesh in Fig. 6. The cold start simulations from the NCEP FNL analyses generate moderate noise from the initial 

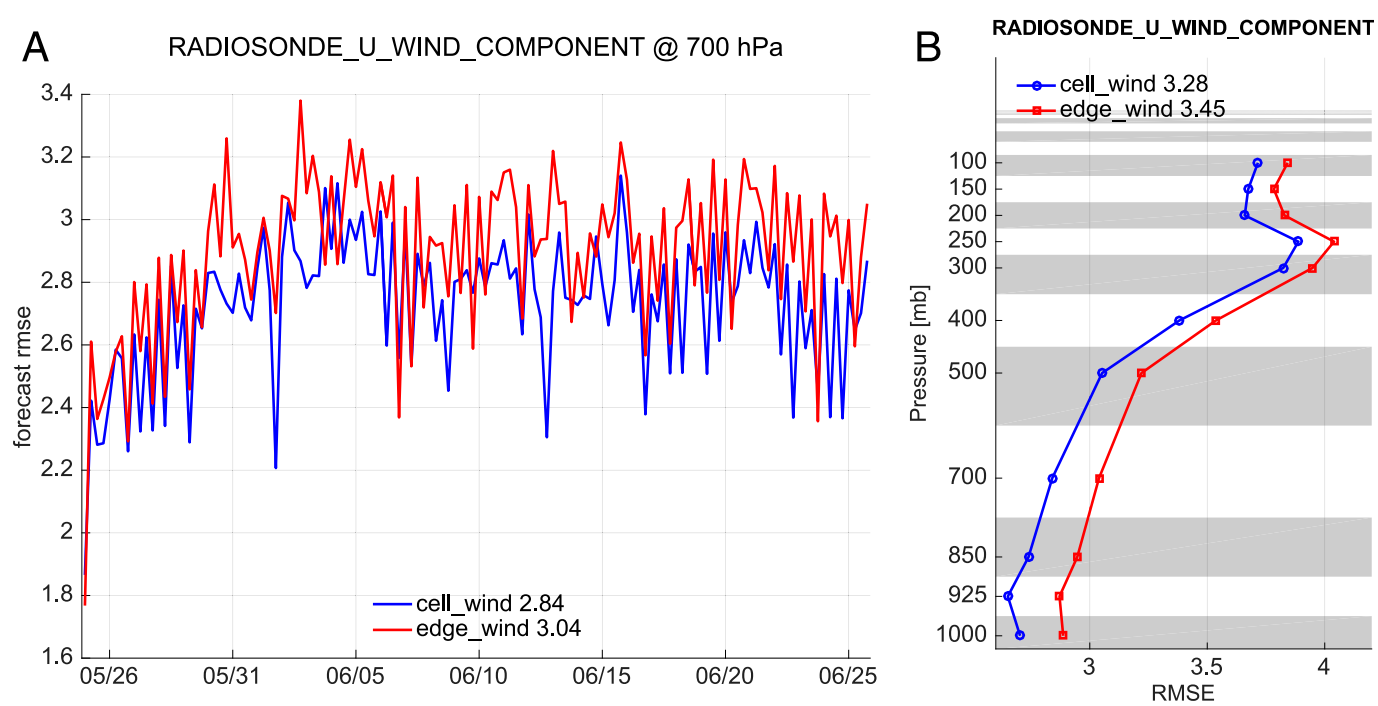

FIG. 5. Root-mean-square errors (rmse) of 6-h ensemble mean forecast in two experiments with different observation operators for horizontal winds, verified against radiosonde zonal wind observations over the globe, are shown in (a) a time series at $700 \mathrm{hPa}$ and (b) the vertical profile (averaged for the cycling period). The mean values over the cycles [in (a)] and over the vertical profile [in (b)] are written next to the experiment names.

time (green line). In the forecasts from the EnKF mean analyses without IAU (black), however, the noise is initially large due to the imbalances in the ensemble analysis, and then quickly drops for the first 5 time steps. Afterward, the noise is only slightly reduced, and mostly remains for 6 -h forecast. With IAU, the forecast starts from $t=-3 \mathrm{~h}$, distributing analysis increments for $6 \mathrm{~h}$ centered on the analysis time, followed by 3-h free forecast, as described in section $2 \mathrm{~d}$. The use of IAU during the cycles suppresses the noise throughout the forecast time, which turns out to be as effective as the cold-start simulation. In our cycling experiments, 6-h forecasts (or 9-h forecasts with IAU) are used as background (or prior) for the next cycle, thus the highfrequency noise at $t=6 \mathrm{~h}$ can contribute to the forecast error growth and the quality of analysis (which is updated based on the background forecasts) during cycles. The variable mesh also shows a very similar trend for both cases with and without IAU (not shown). As the time series pattern and the relative magnitudes of the noise are consistent throughout the cycles, only one sample cycle is presented here.

To examine the effect of the noise reduction on cycling experiments, we make a comparison of prior ensemble mean forecast verification against surface altimeter observations (Fig. 7). As the IAU needs a longer forecast length to run from the beginning of the IAU window (i.e., $9 \mathrm{~h}$ instead of a 6-h forecast to start $3 \mathrm{~h}$ before the analysis time) in each ensemble member every cycle, it requires $30 \%$ more computation time in total. To make a good use of such excessive computational resources, we investigate which one is more effective between the IAU and a larger ensemble size. The default experiment ("e96") uses a 96-member ensemble, the "e192" experiment employs 192 members (without IAU), and "e96_IAU" is the same as "e96" but with IAU during the cycles. Here the prior forecasts are verified against common observations between

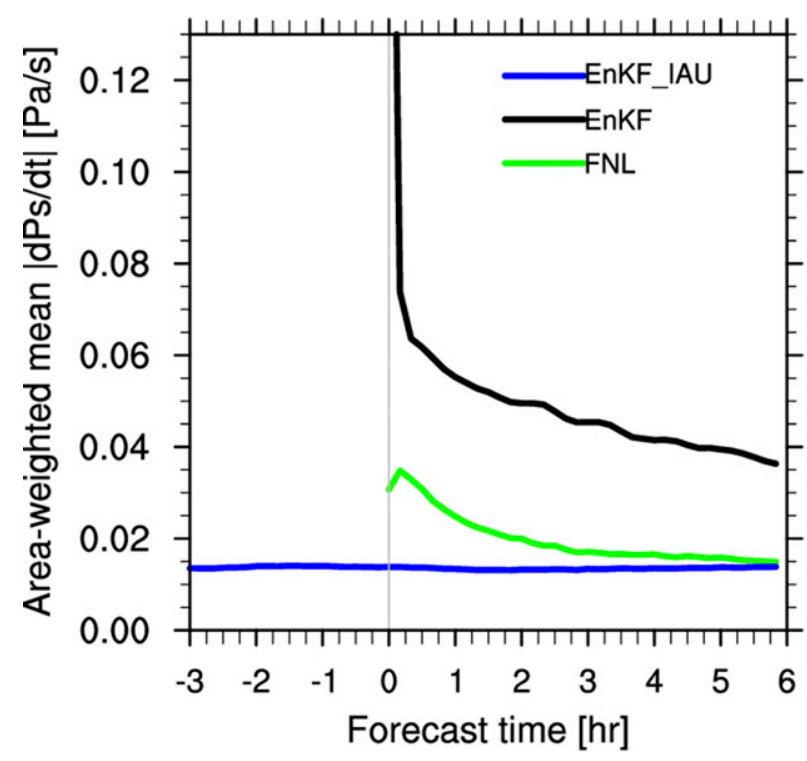

FIG. 6. Area-weighted global mean absolute surface pressure tendencies at each time step for MPAS forecasts from the analyses valid at 1200 UTC 11 Jun 2012. The forecasts from the EnKF analyses with IAU on or off are compared over the uniform (x1) mesh. The MPAS forecasts from the NCEP FNL analyses are marked in green. 

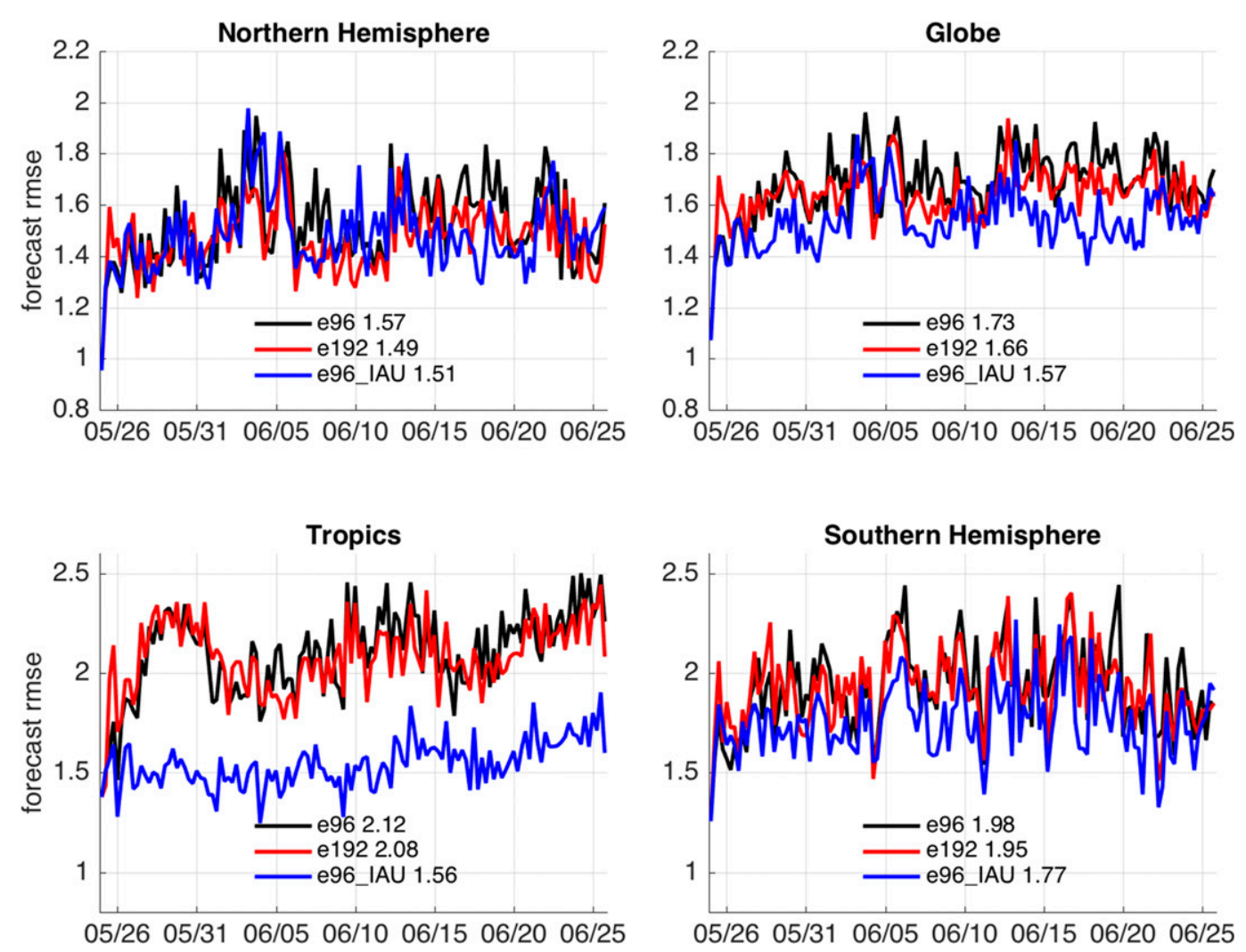

FIG. 7. Time series of 6-h ensemble mean forecast rms fit to surface altimeter settings during the cycles, over four different regions. The default 96-member ensemble ("e96" in black) is compared to a large ensemble run ("e192" in red) and the same 96-member ensemble but with IAU ("e96_IAU" in blue) over the 120-km uniform mesh.

the three experiments. During the cycles, rms errors are largely overlapping, especially in the Northern Hemisphere. Over the tropics, however, the IAU drastically reduces the error for the entire cycling period, being significantly more effective than the large ensemble experiment. In this particular region, the model-minusobservation bias is consistently reduced by a factor of 2 when the IAU is used (not shown). Thus, the forecast improvement by IAU is mostly attributed to the reduction of the systematic error over the tropics. Overall, doubling the ensemble size is not as effective as the IAU in this particular case. It should be noted, however, that to be consistent with all other experiments the same model and filter configurations are used in "e192" except for the ensemble size, which might not maximize the effect of the large ensemble run (e.g., in terms of localization).

\section{Benefit of the variable-resolution mesh}

\section{a. Free forecasts}

The performance of the ensemble analysis relies on the quality of background ensemble forecasts that are used to compute the background error covariance. It is thus important to investigate how the MPAS model behaves, especially in the skill of MPAS forecasts on the variable mesh relative to the ones over the uniform meshes, before comparing cycling experiments for our own analysis. Figure 8 compares the anomaly correlation coefficient (ACC) of geopotential height at $500 \mathrm{hPa}$ over the Northern Hemisphere (NH) for MPAS forecasts over three different meshes. The 5-day forecasts are initialized from the half-degree NCEP FNL analyses for 1-25 June 2012, twice daily, every day, to make a total of 50 samples. The forecast skill of the $120-30-\mathrm{km}$ variable mesh (red) lies between the ones for 30- (blue) and $120-\mathrm{km}$ (orange) uniform meshes, representing the benefit of local refinements that cover about one-third of the verification area. After the 84-h forecast, however, the variable mesh gets closer to the coarse uniform mesh in ACC because the flow becomes gradually dominated by the large-scale features simulated over the coarse mesh area. The ACCs in other regions show that the variable mesh is almost the same as the coarse uniform mesh because simulations are basically done at the same $120-\mathrm{km}$ resolution. Because of the small sample size, ACC values in different meshes are not significantly 


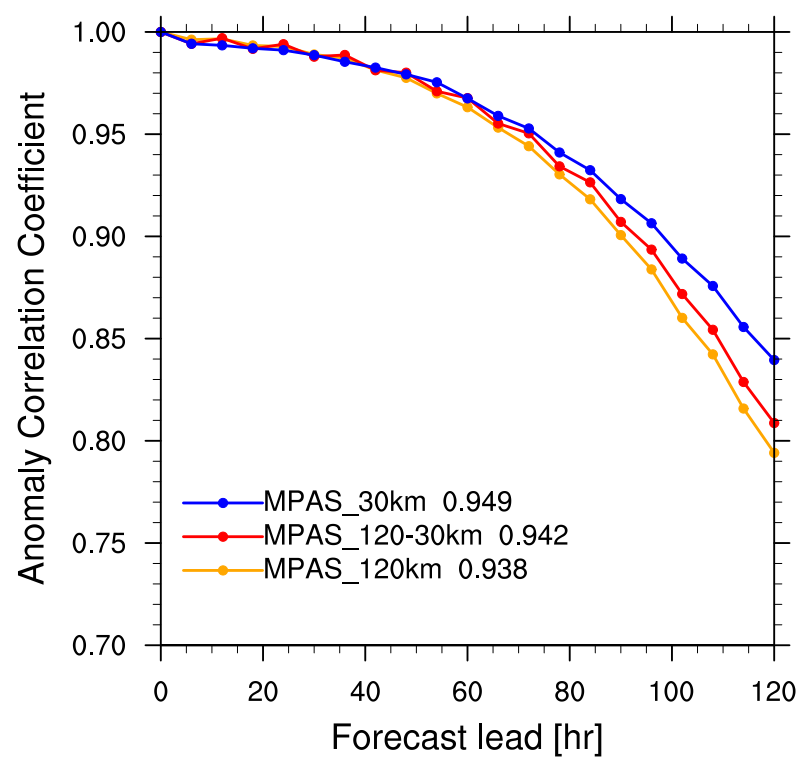

FIG. 8. ACC of geopotential height at $500 \mathrm{hPa}$ over the Northern Hemisphere (NH), for MPAS forecasts on different resolution meshes. The ACC is computed as a function of forecast lead time, initialized from the same $0.5^{\circ} \times 0.5^{\circ} \mathrm{FNL}$ analysis, for a total of 50 samples (for 1-25 Jun 2012, twice daily, every day). The averages over all the lead times are written for each mesh.

different in a statistical sense, but it is confirmed that the MPAS model behaves as expected. Specifically, the error in the fine-mesh region will ultimately be dominated by the coarse-mesh error as has been observed in global MPAS simulations for idealized shallow-water equations tests (Ringler et al. 2011), and as suggested in full atmospheric model tests (e.g., Rauscher et al. 2013).

\section{b. 6-h ensemble forecasts during the cycling}

From the ensemble cycling perspective, it is of the highest priority to make sure that the simulations on the variable mesh remain robust and reliable during the cycling period, and the quality of 6-h forecasts is critical because it is used as the background of the analysis during the 6-h cycling. Figure 9 compares cycling results on the $120-\mathrm{km}$ uniform (x1) and the $120-30-\mathrm{km}$ variable (x4) meshes in 6-h ensemble mean forecast error with respect to surface altimeter observations. Here, we turn off IAU in the assimilation cycle to demonstrate that the cycling experiments are reliable and perform reasonably well in both meshes even without the aid of IAU. The rms error over the variable mesh does not significantly increase or decrease with cycles, and largely overlaps with that in the coarse uniform mesh over the same coarse mesh region (e.g., Southern Hemisphere). But as confirmed in the temporal averages over the cycling period, the variable mesh performs slightly better than the coarse uniform mesh everywhere. Because of computational limitations, we have not conducted an ensemble cycling experiment at global $30-\mathrm{km}$ resolution, but the comparison between the two meshes in the cycling experiments gives results consistent with MPAS free forecasts from the FNL analyses (shown in Fig. 8).

In the DA cycling, it is common to check the observationminus-forecast $(o-f)$ statistics to see if the improvements are made through the analysis. But in this particular mesh comparison (x1 vs $\mathrm{x} 4)$, the $(o-f)$ s are not statistically distinguishable in most fields, thus we focus on the extended forecasts from the EnKF mean analyses on the two meshes, as illustrated in the following sections.

\section{c. Extended forecasts}

To examine the performance of 5-day forecasts, we extend the MPAS forecasts from the EnKF mean analyses from 28 May to 25 June 2012, twice daily (at 0000 and 1200 UTC), every other day, to make a total of 30 samples, and verify them against the NCEP FNL analyses. While the $(o-f)$ s during cycles are very similar between the two meshes with or without IAU, 5-day forecasts from their EnKF mean analyses produce larger differences when the IAU is used during the assimilation cycle. Since the use of IAU produces better forecasts, especially in the tropics, the results presented here are based on that configuration. Effects of omitting IAU are briefly summarized later in this subsection.

Figure 10 compares the time series of rms error in temperature at $500 \mathrm{hPa}$ for 5 -day forecasts using the two meshes. As a baseline, MPAS cold-start simulations initialized from the FNL analyses ${ }^{3}$ are also presented. The cold-start forecasts isolate the effect of using the variable mesh in the forecast model (by using the same analyses on both meshes). They show no error at the initial time since all the forecasts are verified against the FNL analyses. Note that the EnKF analysis is produced on the same meshes as in the forecast model throughout the cycling while the half-degree FNL analysis is initialized by an interpolation to the corresponding meshes and has no extra information for the high-resolution part.

In cold-start simulations from the FNL analysis, the variable mesh produces better forecasts and slower growth of forecast error than the coarse uniform mesh over the CONUS domain (Fig. 10a, cf. orange and green dashed curves). This demonstrates that locally enhancing resolution in the forecast model with the variable mesh improves the forecasts.

\footnotetext{
${ }^{3}$ The FNL analyses include many more observations (especially radiances from various satellite-borne instruments) than our EnKF analyses, and thus can be expected to yield better forecasts, especially over the Southern Hemisphere.
} 


\section{LAND_SFC_ALTIMETER @ 1 surface}
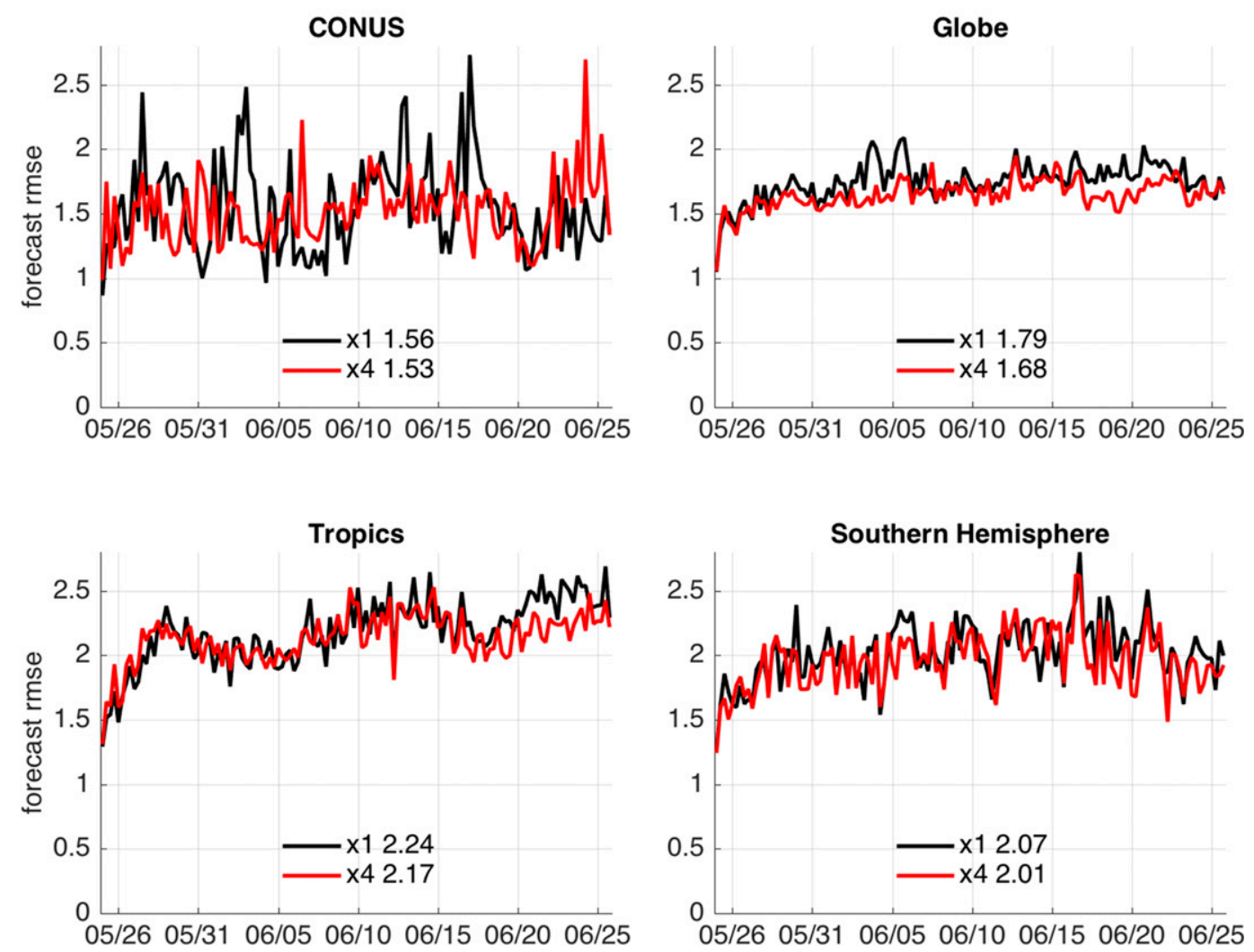

FIG. 9. As in Fig. 7, but for the comparison between two different meshes, verified against common observations between the two experiments. Here, $\mathrm{x} 1$ means the $120-\mathrm{km}$ quasi-uniform mesh (same as "e96" in Fig. 7) while $\mathrm{x} 4$ is the $120-30-\mathrm{km}$ variable-resolution mesh with the entire CONUS domain simulated at $30-\mathrm{km}$ resolution. Cyclemean rms errors are also presented in the legend.

More importantly, Fig. 10a also shows that forecasts from the variable-mesh EnKF analyses are substantially better over the CONUS domain than those from the uniform-mesh analyses (cf. red and blue solid curves). Since the improvement of the day- 5 forecasts is nearly twice as large as that observed for the cold-start forecasts (that do not contain finescale features initially), it is clear that the variable-mesh analysis system provides better analyses, in addition to the benefits of the higherresolution forecasts. Meanwhile, forecast errors are statistically indistinguishable between the two meshes over the Southern Hemisphere where both have the same 120-km resolution, regardless of the analyses used as initial conditions (Fig. 10c). [Because forecast error is so much larger in the Southern Hemisphere, the improvement of the variable-mesh (x4_EnKF) over those on the uniform mesh (x1_EnKF) is apparent for the CONUS domain (Fig. 10a) but not for the globe (Fig. 10b).] Thus, the variable-mesh assimilation system offers significant benefits and no obvious drawbacks, other than increased computational cost, relative to that using the uniform mesh.

Over the tropics, irrespective of the meshes, MPAS forecasts from the EnKF analyses show much slower error growth rates than those from the FNL analyses, leading to better forecasts after $84 \mathrm{~h}$. This is related to the analysis improvements with IAU in this region, as we will discuss further below.

Improvements from the variable mesh are also apparent at other levels and in other variables. Figure 11 shows vertical profiles of the rms and mean temperature forecast errors over the CONUS for day- 5 forecasts. The locally higher-resolution forecasts reduce rms errors throughout the troposphere, particularly between 250 and $925 \mathrm{hPa}$ (Fig. 11a). Use of the variable mesh in the EnKF analysis also significantly reduces the systematic bias relative to the uniform mesh, with reductions of up to $0.8 \mathrm{~K}$ between 300 and $400 \mathrm{hPa}$. In these vertical profiles, the benefit of the variable mesh in the EnKF analysis is much larger than that in the FNL analysis 

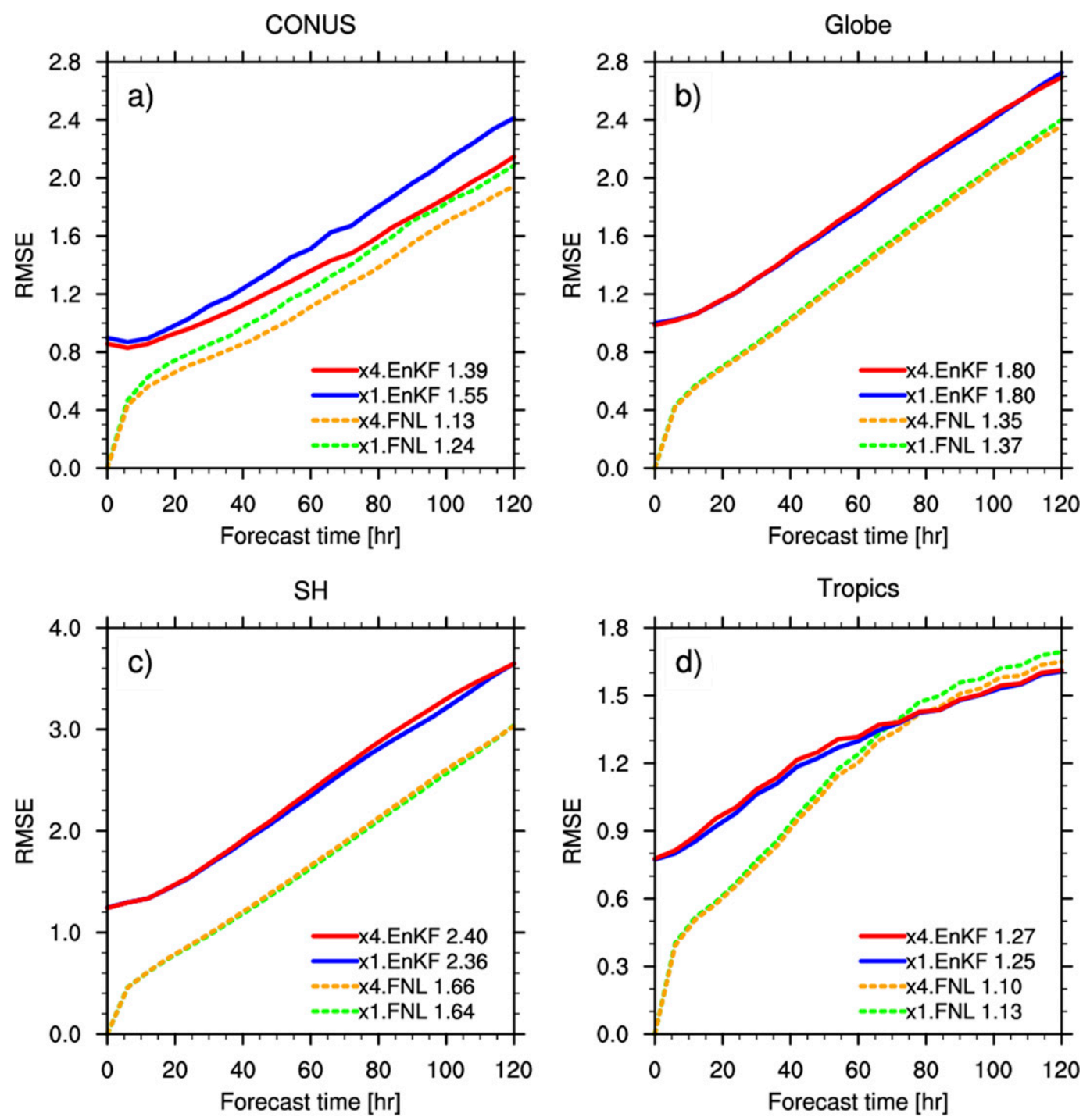

FIG. 10. Verification of MPAS forecasts from different analyses in terms of temperature at $500 \mathrm{hPa}$, with respect to the NCEP FNL analyses, over the (a) CONUS, (b) globe, (c) Southern Hemisphere, and (d) tropics. The rms errors are computed from 28 May to 25 Jun 2012, twice daily (at 0000 and 1200 UTC), every other day. The error averaged over 5-day forecasts is shown for each experiment.

throughout the midtroposphere. Statistics for rms errors are summarized for zonal wind and height, in addition to temperature, at three different isobaric levels in Table 1. They are statistically different between the two meshes up to $90 \%$ significance level in the Student's $t$ test, except for zonal wind.

The effect of IAU on 5-day forecasts is twofold. First, regardless of meshes, the EnKF analysis error is considerably reduced over tropics, consistent with the surface altimeter verification in the observation space, as depicted in Fig. 7. Without IAU, the analysis error in $500-\mathrm{hPa}$ temperature, for instance, is increased from $\sim 0.8$ to $\sim 1.1 \mathrm{~K}$ in both meshes, leading to day- 5 forecast error as large as $0.1 \mathrm{~K}$ (not shown). Second, over the CONUS domain, while the use of IAU does not produce any sizable differences over the coarse uniform mesh, the forecast error in the variable mesh quickly grows after $84 \mathrm{~h}$ leading to day- 5 forecast errors similar to those from the uniform mesh when the IAU is turned off. Thus, the suppression of high-frequency oscillations seems to affect longer forecasts more significantly, over the variable mesh, in particular.

\section{d. Skillful scales on the variable mesh}

Of particular interest in the use of variable-resolution EnKF analyses is to see if the higher-resolution part of 

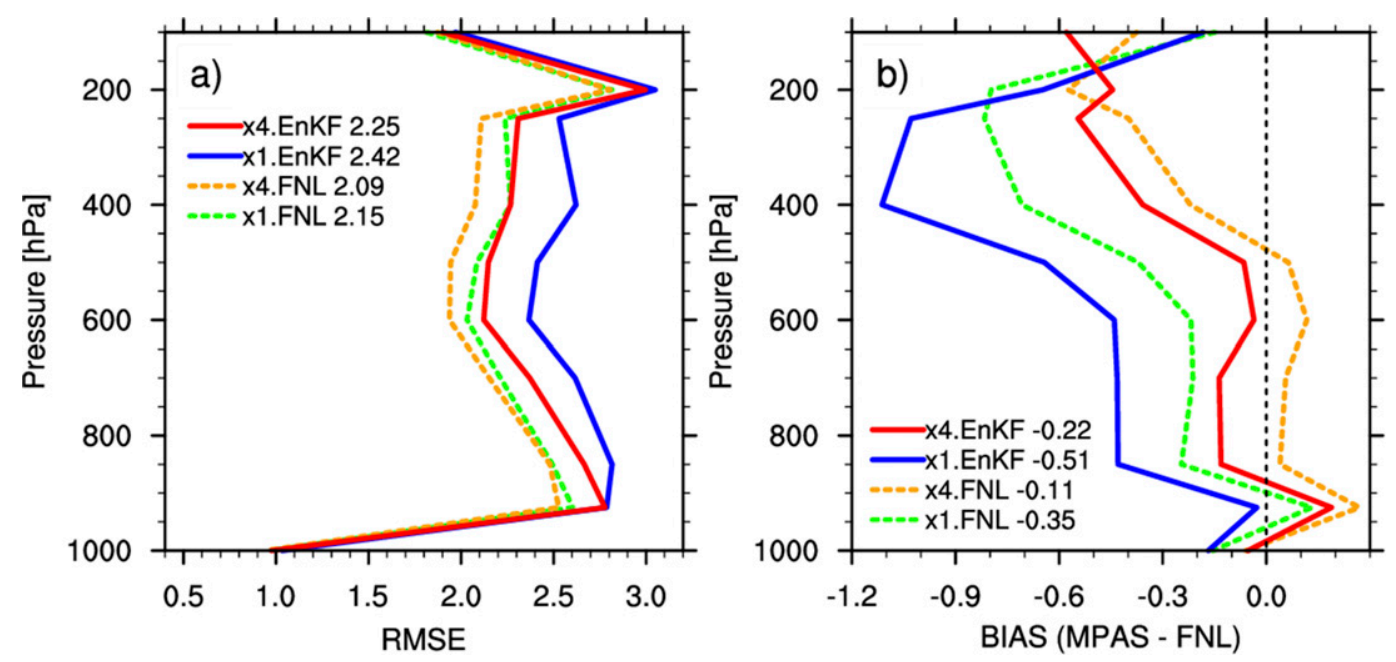

FIG. 11. Vertical profile of day-5 temperature forecast error with respect to the NCEP FNL analysis in (a) rms and (b) bias error over the CONUS domain. Vertically averaged errors are shown in the legend.

the variable mesh can successfully capture mesoscale features in the analysis, and if so, what its effective resolution would be for the mesoscale applications. To gain insight into the model-effective resolution in the analysis during the cycling experiments, we make spectral analyses of ensemble analysis increments in surface pressure in both meshes. To compute spectra, we interpolate the global analysis increments in surface pressure from their native grids into a rectangular latitude-longitude mesh at $0.25^{\circ} \times 0.25^{\circ}$ resolution. Then the interpolated field is analyzed over the CONUS domain extending from $25^{\circ}$ to $55^{\circ} \mathrm{N}$ and from $150^{\circ}$ to $60^{\circ} \mathrm{W}$. For the periodicity in the Fourier transform, the data are first detrended to remove the least squares linear trend along each longitude and tapered with a spatial split-cosinebell function. A Fourier transform is then applied to produce the spectrum. This process is repeated over each ensemble member for 50 sample cycles during the period of 1-25 June 2012, twice daily (0000 and 1200 UTC), every day.

Figure 12 represents the power spectra of surface pressure increments averaged over ensemble members and cycles. All the spectra are truncated at the minimum wavelength resolvable on their native MPAS meshes (i.e., the $2 \Delta x$ wavelength where $\Delta x$ is 30 and $120 \mathrm{~km}$ in $\mathrm{x} 4$ and $\mathrm{x} 1$ meshes, respectively). The spectra of increments in both meshes have almost the same power at the synoptic scale $(>1000 \mathrm{~km})$ and produce a similar slope around their own $6 \Delta$ wavelength (e.g., 180 and $720 \mathrm{~km}$ for $\mathrm{x} 4$ and $\mathrm{x} 1$, respectively). At the smaller scales, however, the analysis increments in the variable mesh have substantially more energy in the mesoscale region. We also checked other variables such as 2-m temperature, upper-level winds, and temperature, in both analysis increments and their full analysis fields, and consistently found the advantage of resolving mesoscale features with more power at the wavelength $<1000 \mathrm{~km}$ in the variable mesh (not shown).

We now focus on precipitation forecasts over the variable mesh $(\mathrm{x} 4)$ to examine the skillful scale in mesoscale simulations from the EnKF analyses over the CONUS domain. Following Roberts (2008) and Roberts and Lean (2008), the fractions skill scores (FSS) are computed over the unstructured mesh to determine the smallest scale, on average, over which the model has useful skill. For that, the fraction of occurrences of specified rainfall accumulations are computed within different-sized sampling areas (neighborhood cells). When $\Delta x$ is a nominal grid spacing in consideration (i.e., $30 \mathrm{~km}$ in the CONUS region), each grid cell is considered as the radius of $1 \Delta x$, and the surrounding neighborhood cells as $2 \Delta x$, and so on. In 6-h cycling experiments, only 6 -h accumulated rainfall is considered for the verification, and 6-hourly accumulations in NCEP Stage-IV data are used as observations. Once the $4-\mathrm{km}$ resolution observations are projected onto the

TABLE 1. The rms errors of MPAS day-5 forecasts verified against NCEP FNL analyses computed over the CONUS domain from 28 May to 25 Jun 2012, twice daily, every other day (making a total of 30 samples). The errors in temperature, zonal wind, and height are compared between 120-km uniform ("x1") and 120 $30-\mathrm{km}$ variable (" $\mathrm{x} 4$ ") meshes at three different isobaric levels.

\begin{tabular}{lccrrrrrr}
\hline \hline & \multicolumn{2}{c}{$T(\mathrm{~K})$} & & \multicolumn{2}{c}{$U\left(\mathrm{~m} \mathrm{~s}^{-1}\right)$} & & \multicolumn{2}{c}{$Z(\mathrm{~m})$} \\
\cline { 2 - 3 } \cline { 9 - 10 } \cline { 8 - 9 } & $\mathrm{x} 1$ & $\mathrm{x} 4$ & & \multicolumn{1}{c}{$\mathrm{x} 1$} & \multicolumn{1}{c}{$\mathrm{x} 4$} & & $\mathrm{x} 1$ & $\mathrm{x} 4$ \\
\hline $250 \mathrm{hPa}$ & 2.53 & 2.31 & & 11.33 & 11.02 & & 70.20 & 63.42 \\
$500 \mathrm{hPa}$ & 2.41 & 2.15 & & 6.78 & 6.73 & & 42.11 & 41.84 \\
$850 \mathrm{hPa}$ & 2.82 & 2.67 & & 4.91 & 4.91 & & 28.24 & 28.65 \\
\hline
\end{tabular}




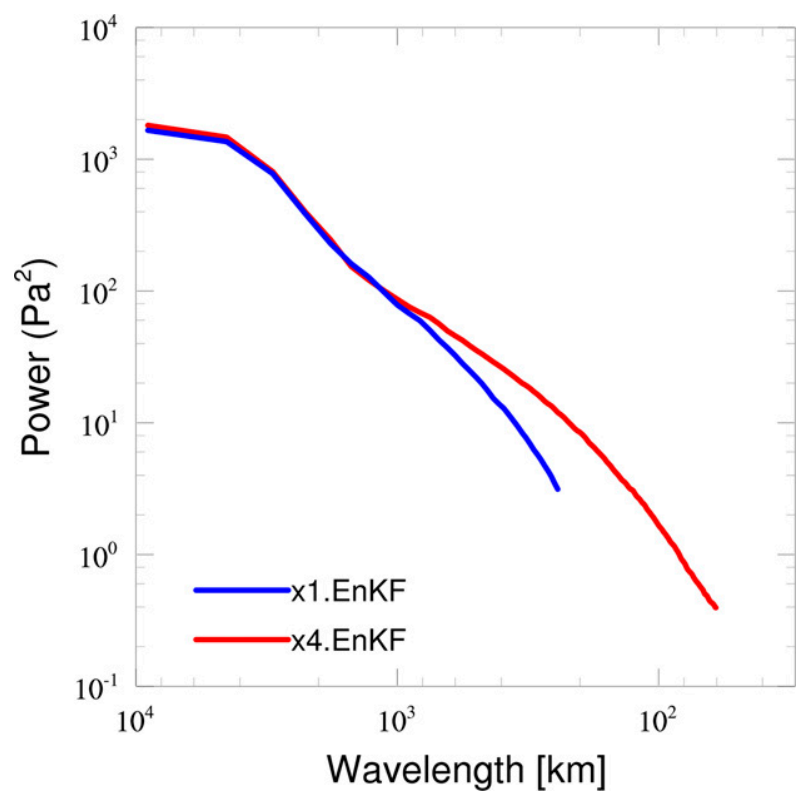

FIG. 12. Spectra of ensemble analysis increments in surface pressure in both the $120-\mathrm{km}$ uniform mesh ("x1.EnKF") and the 120-30-km variable mesh ("x4.EnKF"), averaged over 96-ensemble members and a total of 50 cycles during 1-25 Jun 2012 (twice daily, every day) over the CONUS domain.

variable mesh over the $30-\mathrm{km}$ resolution area, observation and model fractions exceeding thresholds are computed over a range of spatial scales $(n \Delta x)$. In this investigation, percentile rather than accumulation thresholds are presented to focus on the spatial accuracy of precipitation forecasts removing the impact of the bias in rainfall amounts, as in Roberts and Lean (2008).

Figure 13 presents ensemble forecast skills during the cycling experiment, as a function of horizontal scale in kilometers, for the 85th, 90th, 95th, and 99th percentile thresholds. The 85 th percentile represents more widespread areas of weak rain and the 99th percentile more localized and rare events. On average, the thresholds are equivalent to accumulation thresholds of about $0.5,1.0$, 3.0 , and $13 \mathrm{~mm}$ over this particular summer month. Skill is lowest at the grid scale (i.e., $30 \mathrm{~km}$ ) where the fractions are binary ones or zeros, and then improves with spatial scale for all four thresholds. In a large domain (typically with a small wet ratio), forecasts can be considered skillful if FSS goes above 0.5 (Mittermaier et al. 2013). Thus, the smallest scale at which the model has useful skill can be found by the first intercept at the reference value of 0.5 . For example, 6-h MPAS forecast represents a lower bound of useful scales as $5 \Delta x$ $(\sim 150 \mathrm{~km})$ for the $95 \mathrm{th}$ percentile and $14 \Delta x(\sim 420 \mathrm{~km})$ for the 99th percentile. In 6-h ensemble forecast, FSS has larger variabilities across members (e.g., thicker lines) at higher thresholds, as the localized events are

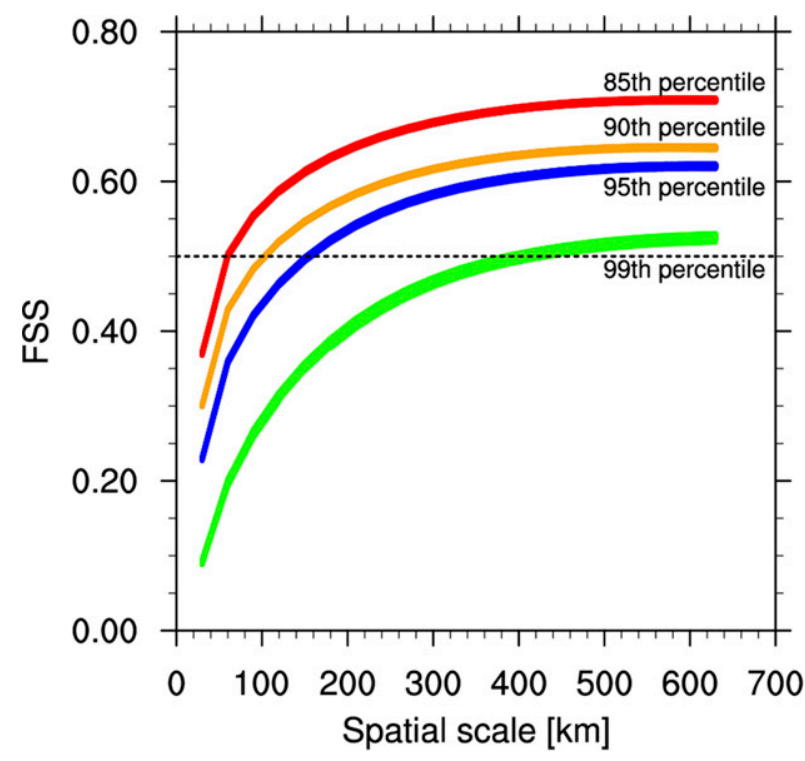

FIG. 13. FSS of 6-h ensemble forecasts from the EnKF analyses on the variable mesh (x4), as a function of horizontal scale for different percentile thresholds, over the CONUS domain. For each percentile 96 ensemble members are drawn on top of each other, and the horizontal dashed line at FSS $=0.5$ represents the reference level of skillful forecasts.

more uncertain. Within the radius of $420 \mathrm{~km}$, for example, ensemble spread at the 99th percentile is almost double the ones at the other three percentile thresholds.

FSS is also computed for extended forecasts from the EnKF mean analysis during cycles-twice daily, every day for 1-25 June 2012 to make total of 50 samples. Comparing Figs. 13 and 14a shows that the MPAS forecast skill quickly gets worse with increasing thresholds in 12-h forecast. For example, the smallest skillful scale for the 85th percentile is almost the same between 6-h ensemble and 12-h deterministic forecasts, but expands from $150 \mathrm{~km}(\sim 5 \Delta x)$ in 6-h forecast to about $210 \mathrm{~km}(\sim 7 \Delta x)$ in 12 -h forecast at the 95th percentile. Because of a small sample size for very localized rainfall events, MPAS cannot make skillful forecasts at $12 \mathrm{~h}$ for the 99 th percentile in this $30-\mathrm{km}$ resolution area regardless of horizontal scale.

Figure $14 \mathrm{~b}$ shows the change of the smallest skillful scale as a function of forecast lead. The scale increases with lead time for all three thresholds, as expected. Because of the sampling error, there are temporal variations in terms of skillful forecast scales, which becomes more evident with heavier rainfall events specified as higher percentile thresholds. Also, the slope gets steeper with higher thresholds, implying that forecasts of more localized heavy rainfall events lose skill more quickly with time. Overall, the variable-mesh MPAS simulations from the EnKF analyses make skillful precipitation 

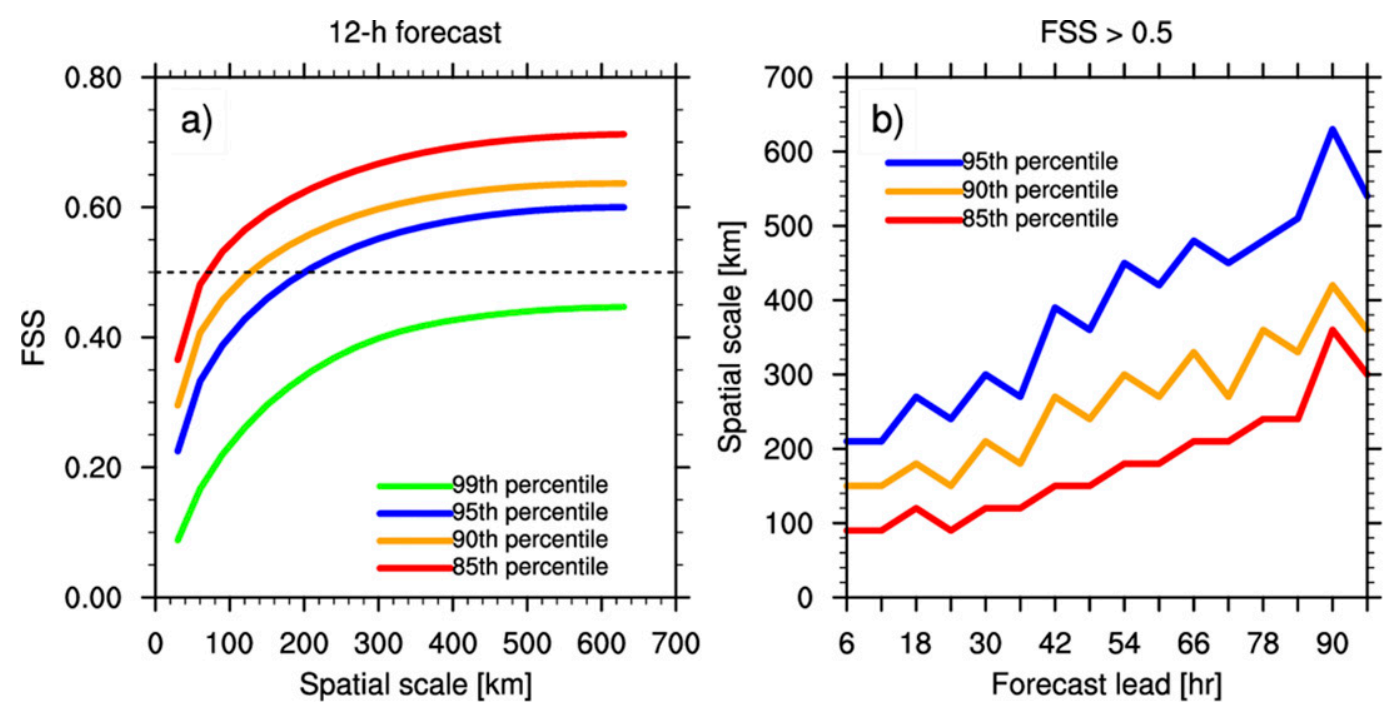

FIG. 14. (a) FSS of MPAS extended forecasts from the EnKF mean analyses as a function of spatial scale at 12-h forecast and (b) the spatial scale at which forecasts become useful (FSS $>0.5$ ) as a function of forecast leads for the 85 th, 90th, and 95th percentile.

forecasts at the scales between $3 \Delta x$ and $21 \Delta x$ depending on the target time and the threshold values.

Figure 15 illustrates the performance of precipitation forecasts over the two different meshes. For this comparison, 5-day forecasts from the coarse uniform mesh (x1) are first projected onto the variable mesh (x4). Considering the representative scale of the original $120-\mathrm{km}$ mesh, the FSS is compared within the radius of $600 \mathrm{~km}$ (e.g., $5 \Delta x$ ) for three different percentile thresholds over the CONUS domain. While the variable mesh is skillful for most of the forecast leads irrespective of thresholds, the uniform mesh quickly loses forecast skill with increasing percentile to become unskillful for heavy rain events (e.g., 95th percentile) after 2-day forecasts. Consistent with the results for other fields displayed in the previous subsections, the variable mesh $(\mathrm{x} 4)$ outperforms the uniform mesh $(\mathrm{x} 1)$ at all three thresholds throughout 5-day forecasts.

\section{Summary and discussion}

This paper introduces a global ensemble data assimilation system that uses the MPAS forecast model and the DART EnKF analysis system. The MPAS-DART system uses the horizontal and vertical mesh of the MPAS model and as such it is immediately applicable for variable-resolution or various grid resolution meshes to facilitate multiscale global ensemble analysis and forecasting. Assimilating the same conventional observations, satellite winds, and GPS radio occultation data, a 96-member global ensemble system using MPAS-DART is cycled over 2 different MPAS meshes, a $120-\mathrm{km}$ quasi-uniform mesh and a $120-30 \mathrm{~km}$ variable-resolution mesh, for a 1-month period.

During the month of cycling, the assimilation system remains robust and reliable on both the quasi-uniform and variable-resolution meshes, but the quality of the short-range forecasts (as measured by the observationforecast differences in the assimilation cycle) are not statistically distinguishable between the two meshes. The benefits of variable-resolution analyses are manifested in 5-day forecasts for a one-month period. In cold-start simulations initialized from the FNL analysis, the variable mesh produces better forecasts and slower error growth than the coarse uniform mesh over the CONUS domain, showing the advantage of locally enhanced resolution in the forecast model. The improvements are even more evident in the warm-start simulations from our own EnKF analyses, where the use of the variable mesh leads to substantially better forecasts than those using the coarse uniform mesh. The improvement in day- 5 forecasts from the EnKF analyses is nearly twice as large as that realized for the cold-start simulations. This demonstrates that the variable-mesh assimilation system provides higher-quality analyses in addition to the benefits of the higher-resolution forecasts. Meanwhile, forecast errors are statistically indistinguishable between the two meshes over the Southern Hemisphere, where both meshes have the same $120-\mathrm{km}$ resolution. This implies that the variable-mesh assimilation system takes advantage of the local mesh refinements while not deteriorating forecasts elsewhere.

We have examined how the variable mesh alters the scales usefully resolved in the analyses and forecasts. 

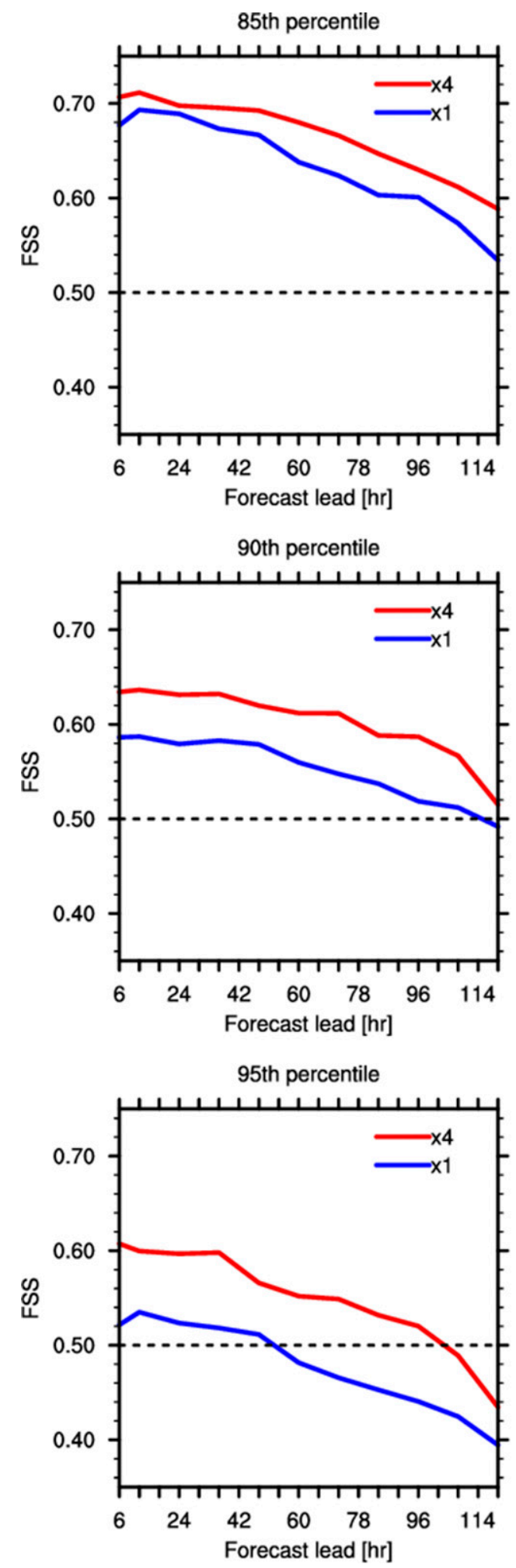

FIG. 15. FSS of 5-day MPAS forecasts from the EnKF mean analyses over 120-km ("x1") and 120-30 km ("x4") meshes within the radius of $600 \mathrm{~km}$, computed for the (top) 85th, (middle) 90th, and (bottom) 95th percentile.
Spectra from surface pressure analysis increments indicate that the EnKF analyses over the variable mesh can resolve shorter scales than those from the coarse uniform mesh, having substantially more energy in the mesoscale range. The spatial scale for sufficiently skillful precipitation forecasts is also examined in the local refinement area over the variable mesh. Computing FSS directly from the unstructured grids, 6-h ensemble forecasts during the EnKF cycling exhibit a smallest skillful scale around $150 \mathrm{~km}$ ( $\sim 5$ grid length) for the $95 \mathrm{th}$ percentile [which corresponds to $3 \mathrm{~mm}(6 \mathrm{~h})^{-1}$ on average], increasing to $420 \mathrm{~km}$ ( $\sim 14$ grid length) for the $99 \mathrm{th}$ percentile $\left[\sim 13 \mathrm{~mm}(6 \mathrm{~h})^{-1}\right]$. The evolution with forecast time is also investigated in the deterministic forecasts from the EnKF ensemble mean analyses. Generally, forecast skill is lost more rapidly at smaller scales and for more localized heavy rainfall events. Comparing to the uniform mesh (regridded from the original $120-\mathrm{km}$ to the same $30-\mathrm{km}$ resolutions over the CONUS), the variable mesh configuration consistently outperforms the coarse uniform mesh configuration in precipitation forecasts at all thresholds and lead times.

These MPAS-DART results are based on the analysis system that utilizes the native MPAS unstructured Voronoi C-grid staggered mesh. DART makes use of barycentric interpolation of MPAS cell-centered scalar variables, based on the dual (triangular) mesh. Cellcentered horizontal zonal and meridional velocities are reconstructed from the MPAS C-grid staggered horizontal velocities, and they are used as the analysis variables in DART. The resulting horizontal velocity increments are projected back to the cell edges to complete the analysis. This approach for handling the horizontal velocity is found to be superior to other possibilities, and it is consistent with how the model physics handles them.

We have also implemented incremental analysis updates (IAU) into MPAS-DART, and shown that the IAU effectively suppresses spurious high-frequency waves and improves the EnKF analysis, particularly in the tropics.

With the encouraging results presented here, there are several areas where development and testing are under way to further improve the system. First, the current MPAS global simulations may be limited by the mesoscale physics suite adopted from the regional WRF Model. For instance, there are regions with large model error, such as near the model top. We leave systematic errors for the future study, and eventually need to improve physics parameterization schemes directly. Accounting for model uncertainty is also important and can be achieved, in part, through stochastic parameterizations in ensemble forecasting (Ha et al. 2015). For 
variable-resolution meshes that reach convectionpermitting resolutions, scale-aware parameterizations will be necessary. A new convection-permitting physics suite is now released in MPAS, version 5, along with the mesoscale_reference suite, and will be tested in the ensemble cycling context in the near future. From the data assimilation point of view, the assimilation of satellite radiances is a critical factor for a high-quality global analysis, especially over the Southern Hemisphere. There are ongoing efforts on incorporating satellite radiance observations into the MPAS-DART system in an efficient way. Finally, more sophisticated strategies for optimized or scale-dependent covariance localization might be needed for multiscale EnKF analyses, particularly over the variable mesh with locally convection-permitting resolution. As of May 2017, the "Manhattan" version of DART is released with an interface to MPAS-A with full capabilities available for use by research community, greatly improving the computational efficiency for large ensemble simulations at high resolutions.

Acknowledgments. We thank Joe Klemp for helpful discussions and his contribution to improving our IAU implementation. This work could not have been done without the support of Michael Duda, Laura Fowler in our Mesoscale and Microscale Meteorology (MMM) laboratory, and Tim Hoar and Kevin Raeder in the DART team. We are also grateful for the support from Mary Haley and Dennis Shea for postprocessing with NCL scripts. The experiments were performed on the Yellowstone supercomputer through the National Center for Atmospheric Research Strategic Capability (NSC) project. This material is based upon work supported by the National Science Foundation under Grant M0856145.

\section{REFERENCES}

Anderson, J. L., 2001: An ensemble adjustment Kalman filter for data assimilation. Mon. Wea. Rev., 129, 2884-2903, https:// doi.org/10.1175/1520-0493(2001)129<2884:AEAKFF>2.0.CO;2.

- 2007 : An adaptive covariance inflation error correction algorithm for ensemble filters. Tellus, 59A, 210-224, https:// doi.org/10.1111/j.1600-0870.2006.00216.x.

__, 2009: Spatially and temporally varying adaptive covariance inflation for ensemble filters. Tellus, $\mathbf{6 1 A}, 72-83$, https://doi.org/10.1111/j.1600-0870.2008.00361.x.

_ 2012: Localization and sampling error correction in ensemble Kalman filter data assimilation. Mon. Wea. Rev., 140, 23592371, https://doi.org/10.1175/MWR-D-11-00013.1.

, T. Hoar, K. Raeder, H. Liu, N. Collins, R. Torn, and A. Avellano, 2009: The Data Assimilation Research Testbed: A community facility. Bull. Amer. Meteor. Soc., 90, 1283-1296, https://doi.org/10.1175/2009BAMS2618.1.
Bloom, S. C., L. L. Takacs, A. M. D. Silva, and D. Ledvina, 1996: Data assimilation using Incremental Analysis Updates. Mon. Wea. Rev., 124, 1256-1271, https://doi.org/10.1175/ 1520-0493(1996)124<1256:DAUIAU>2.0.CO;2.

Bonaventura, L., A. Iske, and E. Miglio, 2011: Kernel-based vector field reconstruction in computational fluid dynamic models. Int. J. Numer. Methods Fluids, 66, 714-729, https://doi.org/ 10.1002/fld.2279.

Buehner, M., and Coauthors, 2015: Implementation of deterministic weather forecasting systems based on ensemblevariational data assimilation at Environment Canada. Part I: The global system. Mon. Wea. Rev., 143, 2532-2559, https:// doi.org/10.1175/MWR-D-14-00354.1.

Côté, J., M. Roch, A. Staniforth, and L. Fillion, 1993: A variableresolution semi-Lagrangian finite-element global model of the shallow-water equations. Mon. Wea. Rev., 121, 231-243, https:// doi.org/10.1175/1520-0493(1993)121<0231:AVRSLF>2.0.CO;2.

Cucurull, L., 2010: Improvement in the use of an operational constellation of GPS radio occultation receivers in weather forecasting. Wea. Forecasting, 25, 749-767, https://doi.org/ 10.1175/2009WAF2222302.1.

Du, J., J. Zhu, F. Fang, C. C. Pain, and I. M. Navon, 2016: Ensemble data assimilation applied to an adaptive mesh ocean model. Int. J. Numer. Methods Fluids, 82, 997-1009, https://doi.org/ 10.1002/fld.4247.

Evensen, G., 1994: Sequential data assimilation with a nonlinear quasi-geostrophic model using Monte Carlo methods to forecast error statistics. J. Geophys. Res., 99, 10143-10162, https://doi.org/10.1029/94JC00572.

Fox-Rabinovitz, M. S., L. L. Takacs, and R. C. Govindaraju, 2002: A variable-resolution stretched-grid general circulation model and data assimilation system with multiple areas of interest: Studying the anomalous regional climate events of 1998. J. Geophys. Res., 107, 4768, https://doi.org/10.1029/2002JD002177.

Gaspari, G., and S. E. Cohn, 1999: Construction of correlation functions in two and three dimensions. Quart. J. Roy. Meteor. Soc., 125, 723-757, https://doi.org/10.1002/qj.49712555417.

Greybush, S. J., E. Kalnay, T. Miyoshi, K. Ide, and B. R. Hunt, 2011: Balance and ensemble Kalman filter localization techniques. Mon. Wea. Rev., 139, 511-522, https://doi.org/10.1175/ 2010MWR3328.1.

Ha, S., J. Berner, and C. Snyder, 2015: A comparison of model error representations in mesoscale ensemble data assimilation. Mon. Wea. Rev., 143, 3893-3911, https://doi.org/10.1175/ MWR-D-14-00395.1.

Hamill, T. M., J. S. Whitaker, and C. Snyder, 2001: Distancedependent filtering of background error covariance estimates in an ensemble Kalman filter. Mon. Wea. Rev., 129, 2776-2790, https://doi.org/10.1175/1520-0493(2001)129<2776: DDFOBE $>2.0 . \mathrm{CO} ; 2$.

Hong, S.-Y., and J.-O. J. Lim, 2006: The WRF single-moment 6-class microphysics scheme (WSM6). J. Korean Meteor. Soc, 42, 129-151.

— Y. Noh, and J. Dudhia, 2006: A new vertical diffusion package with an explicit treatment of entrainment processes. Mon. Wea. Rev., 134, 2318-2341, https://doi.org/10.1175/ MWR3199.1.

Houtekamer, P. L., and H. L. Mitchell, 2001: A sequential ensemble Kalman filter for atmospheric data assimilation. Mon. Wea. Rev., 129, 123-137, https://doi.org/10.1175/ 1520-0493(2001)129<0123:ASEKFF>2.0.CO;2.

- and - 2005: Ensemble Kalman filtering. Quart. J. Roy. Meteor. Soc., 131, 3269-3289, https://doi.org/10.1256/qj.05.135. 
_ , and F. Zhang, 2016: Review of the ensemble Kalman filter for atmospheric data assimilation. Mon. Wea. Rev., 144, 44894532, https://doi.org/10.1175/MWR-D-15-0440.1.

Iacono, M. J., J. S. Delamere, E. J. Mlawer, M. W. Shephard, S. A. Clough, and W. D. Collins, 2008: Radiative forcing by longlived greenhouse gases: Calculations with the AER radiative transfer models. J. Geophys. Res., 113, D13103, doi:10.1029/ 2008JD009944.

Kepert, J. D., 2009: Covariance localisation and balance in an ensemble Kalman filter. Quart. J. Roy. Meteor. Soc., 135, 11571176, https://doi.org/10.1002/qj.443.

Kim, Y.-J., and J. D. Doyle, 2005: Extension of an orographic-drag parameterization scheme to incorporate orographic anisotropy and flow blocking. Quart. J. Roy. Meteor. Soc., 131, 18931921, https://doi.org/10.1256/qj.04.160.

Klemp, J. B., 2011: A terrain-following coordinate with smoothed coordinate surfaces. Mon. Wea. Rev., 139, 2163-2169, https:// doi.org/10.1175/MWR-D-10-05046.1.

Kuo, Y.-H., T.-K. Wee, S. Sokolovskiy, C. Rocken, W. Schreiner, D. Hunt, and R. A. Anthes, 2004: Inversion and error estimation of GPS radio occultation data. J. Meteor. Soc. Japan, 82, 507-531, https://doi.org/10.2151/jmsj.2004.507.

Laroche, S., P. Gauthier, J. St-James, and J. Morneau, 1999: Implementation of a 3D variational data assimilation system at the Canadian Meteorological Centre. Part II: The regional analysis. Atmos.-Ocean, 37, 281-307, https://doi.org/10.1080/ 07055900.1999.9649630.

Lei, L., and J. S. Whitaker, 2016: A four-dimensional incremental analysis update for the ensemble Kalman filter. Mon. Wea. Rev., 144, 2605-2621, https://doi.org/10.1175/MWR-D-15-0246.1.

Lorenc, A. C., N. E. Bowler, A. M. Clayton, and S. R. Pring, 2015: Comparison of Hybrid-4DEnVar and Hybrid-4DVar data assimilation methods for global NWP. Mon. Wea. Rev., 143, 212-229, https://doi.org/10.1175/MWR-D-14-00195.1.

Mitchell, H. L., P. L. Houtekamer, and G. Pellerin, 2002: Ensemble size, balance, and model-error representation in an ensemble Kalman filter. Mon. Wea. Rev., 130, 2791-2808, https://doi.org/ 10.1175/1520-0493(2002)130<2791:ESBAME>2.0.CO;2.

Mittermaier, M., N. Roberts, and S. A. Thompson, 2013: A longterm assessment of precipitation forecast skill using the Fractions Skill Score. Meteor. Appl., 20, 176-186, https:// doi.org/10.1002/met.296.

Monin, A. S., and A. M. Obukhov, 1954: Basic laws of turbulent mixing in the surface layer of the atmosphere (in Russian). Contrib. Geophys. Inst. Acad. Sci. USSR, 151, 163-187.

Nerger, L. S., S. Danilov, G. Kivman, W. Hiller, and J. Schrter, 2007: Data assimilation with the ensemble Kalman filter and the SEIK filter applied to a finite element model of the North Atlantic. J. Mar. Syst., 65, 288-298, https://doi.org/10.1016/ j.jmarsys.2005.06.009.

Peixoto, P. S., and S. R. M. Barros, 2014: On vector field reconstructions for semi-Lagrangian transport methods on geodesic staggered grids. J. Comput. Phys., 273, 185-211, https://doi.org/10.1016/j.jcp.2014.04.043.

Polavarapu, S., S. Ren, A. M. Clayton, D. Sankey, and Y. Rochon, 2004: On the relationship between incremental analysis updating and incremental digital filtering. Mon. Wea. Rev., 132, 2495-2502, https://doi.org/10.1175/1520-0493(2004)132<2495: OTRBIA $>2.0 . \mathrm{CO} ; 2$.
Rauscher, S., T. D. Ringler, W. C. Skamarock, and A. A. Mirin, 2013: Exploring a global multiresolution modeling approach using aquaplanet simulations. J. Climate, 26, 2432-2452, https://doi.org/10.1175/JCLI-D-12-00154.1.

Ringler, T., L. Ju, and M. Gunzburger, 2008: A multiresolution method for climate system modeling: Application of spherical centroidal Voronoi tessellations. Ocean Dyn., 58, 475-498, https://doi.org/10.1007/s10236-008-0157-2.

__ J. J. Thuburn, J. B. Klemp, and W. C. Skamarock, 2010: A unified approach to energy conservation and potential vorticity dynamics for arbitrarily-structured C-grids. J. Comput. Phys., 229, 3065-3090, https://doi.org/10.1016/j.jcp.2009.12.007.

- D. Jacobsen, M. Gunzburger, L. Ju, M. Duda, and W. Skamarock, 2011: Exploring a multiresolution modeling approach within the shallow-water equations. Mon. Wea. Rev., 139, 3348-3368, https://doi.org/10.1175/MWR-D-10-05049.1.

Roberts, N., 2008: Assessing the spatial and temporal variation in the skill of precipitation forecasts from an NWP model. $M e-$ teor. Appl., 15, 163-169, https://doi.org/10.1002/met.57.

— , and H. W. Lean, 2008: Scale-selective verification of rainfall accumulations from high-resolution forecasts of convective events. Mon. Wea. Rev., 136, 78-97, https://doi.org/10.1175/ 2007MWR2123.1.

Skamarock, W. C., and Coauthors, 2008: A description of the Advanced Research WRF version 3. NCAR Tech. Note NCAR/TN-475+STR, 113 pp., http://dx.doi.org/10.5065/ D68S4MVH.

— J. B. Klemp, L. D. Fowler, M. G. Duda, S.-H. Park, and T. D. Ringler, 2012: A multiscale nonhydrostatic atmospheric model using centroidal Voronoi tesselations and C-grid staggering. Mon. Wea. Rev., 140, 3090-3105, https://doi.org/ 10.1175/MWR-D-11-00215.1.

Tewari, M., and Coauthors, 2004: Implementation and verification of the unified Noah land surface model in the WRF model. 20th Conf. on Weather Analysis and Forecasting/16th Conf. on Numerical Weather Prediction, Phoenix, AZ, Amer. Meteor. Soc., 14.2a, https://ams.confex.com/ams/84Annual/ techprogram/paper_69061.htm.

Thuburn, J., T. D. Ringler, W. C. Skamarock, and J. B. Klemp, 2009: Numerical representation of geostrophic modes on arbitrarily structured C-grids. J. Comput. Phys., 228, 8321-8335, https://doi.org/10.1016/j.jcp.2009.08.006.

Tiedtke, M., 1989: A comprehensive mass flux scheme for cumulus parameterization in large-scale models. Mon. Wea. Rev., 117, 1779-1800, https://doi.org/10.1175/1520-0493(1989)117<1779: ACMFSF $>2.0 . \mathrm{CO} ; 2$.

Tippett, M. K., J. L. Anderson, C. H. Bishop, T. M. Hamill, and J. S. Whitaker, 2003: Ensemble square root filters. Mon. Wea. Rev., 131, 1485-1490, https://doi.org/10.1175/1520-0493(2003) $131<1485$ :ESRF $>2.0$. CO;2.

Whitaker, J. S., and T. M. Hamill, 2002: Ensemble data assimilation without perturbed observations. Mon. Wea. Rev., 130, 1913-1924, https://doi.org/10.1175/1520-0493(2002)130<1913: EDAWPO $>2.0 . \mathrm{CO} ; 2$.

Zhu, Y., R. Todling, J. Guo, S. E. Cohn, I. M. Navon, and Y. Yang, 2003: The GEOS-3 retrospective data assimilation system: The 6-hour lag case. Mon. Wea. Rev., 131, 2129-2150, https://doi.org/10.1175/1520-0493(2003)131<2129: TGRDAS $>2.0 . \mathrm{CO} ; 2$. 